\title{
Antisense oligonucleotide-mediated ataxin-1 reduction prolongs survival in SCA1 mice and reveals disease-associated transcriptome profiles
}

\author{
Jillian Friedrich, ${ }^{1,2}$ Holly B. Kordasiewicz, ${ }^{3}$ Brennon O'Callaghan, ${ }^{1,2}$ Hillary P. Handler, ${ }^{1,4}$ \\ Carmen Wagener, ${ }^{1,2}$ Lisa Duvick, ${ }^{1,2}$ Eric E. Swayze, ${ }^{3}$ Orion Rainwater, ${ }^{1,2}$ Bente Hofstra, ${ }^{1,2}$ \\ Michael Benneyworth, ${ }^{5}$ Tessa Nichols-Meade, ${ }^{5}$ Praseuth Yang, ${ }^{1,2}$ Zhao Chen, ${ }^{1,2}$ Judit Perez Ortiz, ${ }^{1,4}$ \\ H. Brent Clark, ${ }^{2}$ Gülin Öz, ${ }^{6}$ Sarah Larson, ${ }^{6}$ Huda Y. Zoghbi, ${ }^{7}$ Christine Henzler, ${ }^{8}$ and Harry T. Orr ${ }^{1,2}$ \\ ${ }^{1}$ Institute for Translational Neuroscience and 'Department of Laboratory Medicine and Pathology, University of Minnesota, \\ Minneapolis, Minnesota, USA. ${ }^{3}$ Ionis Pharmaceuticals, Carlsbad, California, USA. ${ }^{4}$ Graduate Program in Neuroscience, \\ ${ }^{5}$ Department of Neuroscience, and ${ }^{6}$ Center for Magnetic Resonance Research, Department of Radiology, University of \\ Minnesota, Minneapolis, Minnesota, USA. 'Jan and Dan Duncan Neurological Research Institute at Texas Children's \\ Hospital and Department of Molecular and Human Genetics, Howard Hughes Medical Institute, Baylor College of Medicine, \\ Houston, Texas, USA. ${ }^{8}$ Research Informatics Support Systems Bioinformatics, Minnesota Supercomputing Institute, \\ University of Minnesota, Minneapolis, Minnesota, USA
}

Spinocerebellar ataxia type 1 (SCA1) is a dominantly inherited ataxia caused by expansion of a translated CAC repeat encoding a glutamine tract in the ataxin-1 (ATXN1) protein. Despite advances in understanding the pathogenesis of SCA1, there are still no therapies to alter its progressive fatal course. RNA-targeting approaches have improved disease symptoms in preclinical rodent models of several neurological diseases. Here, we investigated the therapeutic capability of an antisense oligonucleotide (ASO) targeting mouse Atxn1 in Atxn 1540/20-knockin mice that manifest motor deficits and premature lethality. Following a single ASO treatment at 5 weeks of age, mice demonstrated rescue of these disease-associated phenotypes. RNA-sequencing analysis of genes with expression restored to WT levels in ASO-treated Atxn $1^{1540 / 20}$ mice was used to demonstrate molecular differences between SCA1 pathogenesis in the cerebellum and disease in the medulla. Finally, select neurochemical abnormalities detected by magnetic resonance spectroscopy in vehicle-treated Atxn $1^{1540 / 20}$ mice were reversed in the cerebellum and brainstem (a region containing the pons and the medulla) of ASO-treated Atxn 1540/20 mice. Together, these findings support the efficacy and therapeutic importance of directly targeting ATXN1 RNA expression as a strategy for treating both motor deficits and lethality in SCA1.

Conflict of interest: HBK and EES have equity in and are employed by lonis Pharmaceuticals, which is developing an ASO-based drug for SCA1.

License: Copyright 2018, American Society for Clinical Investigation.

Submitted: June 26, 2018 Accepted: September 19, 2018 Published: November 2, 2018

\section{Reference information:} JCI Insight. 2018;3(21):e123193. https://doi.org/10.1172/jici. insight.123193.

\section{Introduction}

Spinocerebellar ataxia type 1 (SCA1) is a progressive fatal autosomal dominant neurodegenerative disease caused by a CAG trinucleotide repeat expansion within the coding region of the ataxin-1 (ATXN1) gene (1). Early symptoms typically include gait and limb ataxia, dysarthria, and dysmetria, with disease progressing over 10-15 years, culminating in death from dysphagia and/or respiratory failure secondary to involvement of medullary cranial nerve nuclei and anterior horn neurons (2-4). Accordingly, MRI scans of SCA1 patients reveal severe atrophy of the cerebellum and brainstem (5), and magnetic resonance spectroscopy (MRS) scans show neurochemical abnormalities in the cerebellum and brainstem, which correlate with the clinical status of patients $(6,7)$. Over the years, studies using mouse SCA1 models provided important insight into the cellular and molecular mechanisms underlying SCA1 pathogenesis. Evidence supports the concept that the disease-causing mutation primarily acts through a toxic gainof-function mechanism (8). Importantly, these pathogenic mechanisms are dampened by approaches that reduce expression of ATXN1. Studies using a doxycycline-regulated Pcp2-ATXN1/82Q]-transgenic mouse model of SCA1 demonstrated that repressing mutant protein production 12 weeks after sustained 
expression significantly improved Purkinje cell pathology, behavior deficits (9), and neurochemical abnormalities $(10,11)$. At an early stage of disease (6 weeks of age), Purkinje cell pathology and motor dysfunction were completely reversible. Partial recovery was seen after halting ATXN1 expression at a later stage of disease (12 weeks of age). Thus, at least for the Purkinje cell aspects of SCA1, a window of opportunity exists for gene-silencing strategies initiated after disease onset and before loss of Purkinje cells. ATXN1 RNAi administered by AAV injection into the deep cerebellar nuclei of Pcp2-ATXN1[82Q]transgenic mice also demonstrates effectiveness in reducing $A T X N 1$ expression as a means of alleviating SCA1-like symptoms in mice $(12,13)$. Additional studies using the Atxn 154Q/2Q-knockin mouse model of SCA1 show that even a partial reduction of ATXN1, on the order of $20 \%$, results in detectable improvement in motor performance and cerebellar pathologies $(14,15)$. Importantly, for any strategy targeting the reduction of both WT and mutant ATXN1 alleles, mice lacking Atxn1 show mild learning deficits but do not show signs of ataxia and neurodegeneration (16).

Antisense oligonucleotide-mediated (ASO-mediated) RNA suppression approaches have been used to reduce gene expression and improve disease symptoms in preclinical rodent models of several neurological diseases (17-20), including SCA2 and SCA3 $(21,22)$. There also have been successful clinical trials and FDA approval of an ASO drug that alters SMN2 gene splicing for SMA (23). Therefore, to study therapeutic effects of an ASO-mediated ATXN1 reduction strategy on motor performance and lethality in SCA1, we developed an ASO that selectively targeted mouse Atxn1 RNA throughout the brain. Atxn1 ${ }^{1540 / 20}$ mice, generated by insertion of an expanded CAG repeat into 1 allele of the Atxn1 gene, express ATXN1[154Q] throughout the brain and display 2 key SCA1 phenotypes, ataxia and premature lethality (24). Upon administration of the Atxn1 ASO to Atxn $1^{154 Q / 2 Q}$ mice, motor deficits were rescued and life span was extended. Previously, we utilized cerebellar RNA sequencing (RNA-seq) to define a gene network associated with cerebellar disease progression in an ATXN1[82Q]-transgenic mouse model of SCA1 (25). Here we utilized RNA-seq on the cerebella, pontes, and medullae in vehicle-treated Atxn $1^{1540 / 2 O}$ and ASO-treated Atxn $1^{154 Q / 2 Q}$ mice as means of comparing disease process in regions of the brain severely affected by SCA1. Finally, we utilized cerebellar and brainstem MRS to evaluate its utility to provide noninvasive neurochemical biomarkers to monitor the response to ASO treatment in Atxn $1^{154 Q / 2 Q}$ mice.

\section{Results}

Identification and characterization of the optimal Atxn1 RNA-targeting ASO to reduce Atxn1 expression in regions of the brain. Initial characterization of Atxn1 RNA-targeting ASOs was performed using Atxn166/20 mice. These mice were generated by inserting an expanded CAG tract of 82 repeats into 1 Atxn1 allele (26). With subsequent breeding, we found, by DNA sequence analysis, that the 82 repeats of the $\operatorname{Atxn} 1^{82 Q}$ allele had decreased to 66, thereby resulting in animals harboring the Atxn $1^{660}$ allele. While Atxn $1^{660 / 20}$ mice did not show robust signs of a SCA1-like disease, they allowed assessment of the effects of ASOs on expression of WT and polyQ-expanded Atxn1 alleles in animals that breed well. Three lead ASOs, 20-mer phosphorothioate-modified oligonucleotides designed to target mouse Atxn1 RNA having 2'-O-(-2-methoxy) ethyl modifications on 5 nucleotides on the 3' and 5' ends to increase stability and potency and decrease toxicity (27), were injected into the right lateral ventricle of 5 -week-old Atxn $1^{66 \mathrm{Q} / 2 Q}$ mice ( $500 \mu \mathrm{g}$ in $10 \mu \mathrm{l}$ at $25 \mathrm{nl} / \mathrm{s}$ ). Two weeks after injection, the most substantial reductions of Atxn1 mRNA levels in regions critical for SCA1 pathology, the cerebellum, cerebral cortex, and brainstem, were observed with ASO353 (Supplemental Figure 1A; supplemental material available online with this article; https://doi.org/10.1172/jci.insight.123193DS1).

Next, we assessed the cellular distribution of Atxn1 ASO353 in mouse brains. This was accomplished by administering an i.c.v. bolus injection of Atxn1 ASO353 to 8-week-old Atxn $1^{66 / 20}$ mice and harvesting brains 2 weeks later. Sections were stained for the ASO using an antibody recognizing the ASO backbone chemistry (28). Supplemental Figure 1B shows that the Atxn1 ASO was taken up by neurons throughout the CNS, including the cerebellar cortex, cerebral cortex, and brainstem. Atxn1 ASO353 appeared to be uniformly taken up by neurons in both the cerebral cortex and brainstem (Supplemental Figure 1B). In the cerebellum, Purkinje cells took up Atxn1 ASO353 more robustly than granule cells, the most numerous neuronal type in the cerebellar cortex (Supplemental Figure 1C).

To examine the extent and duration to which ASO353-mediated reduction of Atxn1 RNA and protein endured, a cohort of $\operatorname{Atxn} 1^{66 \mathrm{Q} / 20}$ mice were given a single bolus i.c.v. injection at 5 weeks of age and Atxn1 RNA levels were assessed at 2, 12, and 18 weeks after i.c.v. injection. Figure 1 shows that the reduction in 
- Atxn1 RNA - vehicle

A
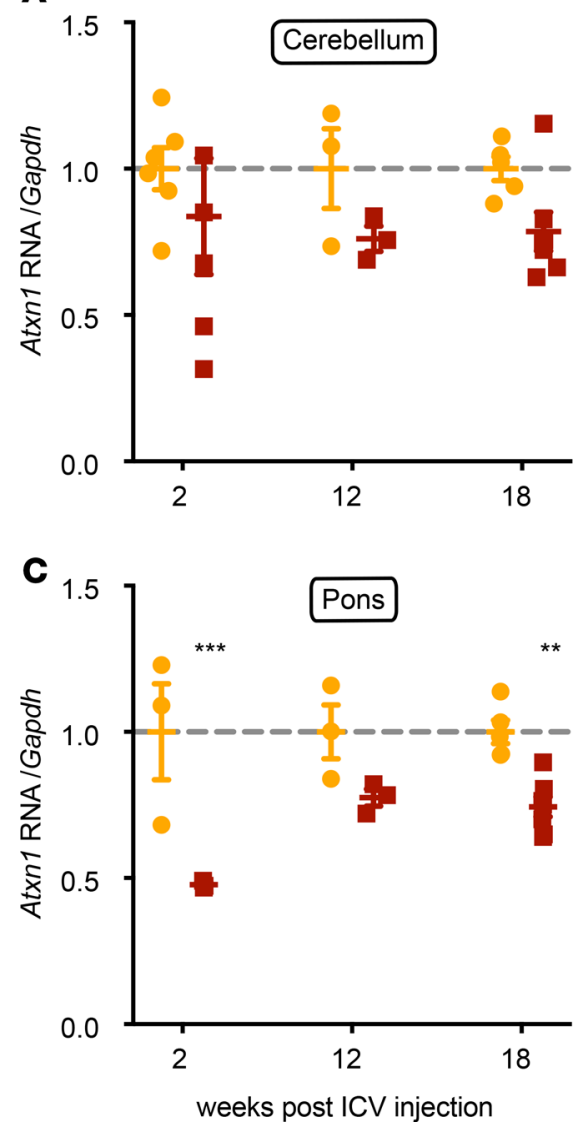

Atxn1 RNA-ASO353

B

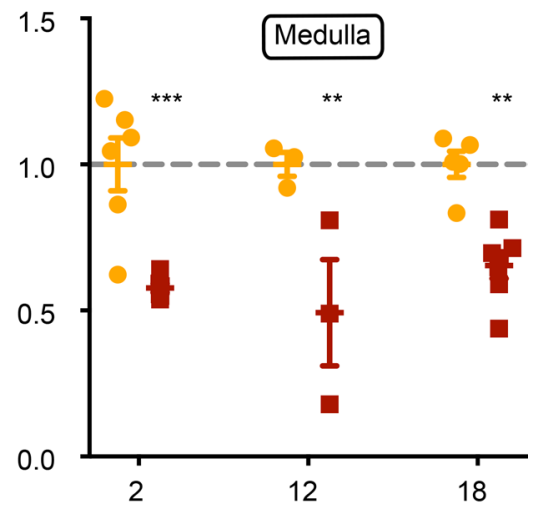

D

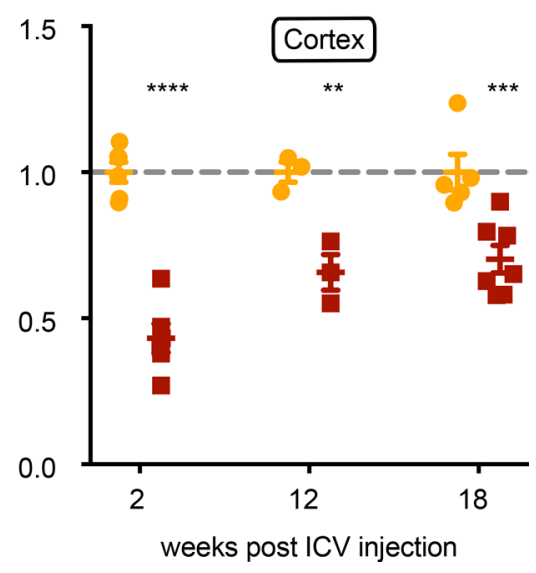

Figure 1. Reduction of Atxn1 RNA by Atxn1 AS0353 in brain regions of Atxn166/20 mice affected by SCA1. $500 \mu \mathrm{g}$ Atxn1 ASO or vehicle was delivered by bolus i.c.v. injection to 5-week-old Atxn ${ }^{660 / 20}$ mice. Atxn1 RNA analyzed by qPCR at 2, 12 and 18 weeks after i.c.v. injection. (A) Atxn1 RNA analyzed from the cerebellum; 2-week ASO and vehicle $(n=6), 12$-week ASO and vehicle $(n=3)$, 18-week ASO ( $n=5)$, 18-week vehicle $(n=7)$. (B) Atxn1 RNA analyzed from the medulla; 2-week ASO and vehicle $(n=6)$, 12-week ASO and vehicle $(n=3)$, 18-week ASO $(n=5)$, 18-week vehicle $(n=7)$. (C) Atxn1 RNA analyzed from the pons; 2 week and 12-week ASO and vehicle $(n=3)$, 18-week ASO $(n=5)$, 18-week vehicle ( $n=7)$. (D) Atxn1 RNA analyzed from the cerebral cortex; 2-week ASO and vehicle $(n=6), 12$-week ASO and vehicle $(n=3)$, 18-week ASO $(n=5)$, 18 -week vehicle $(n=7)$. Data are presented as mean \pm SEM. ${ }^{* *} P<0.01,{ }^{* *} P<0.001$, ${ }^{* * *} P<0.0001,2$-way ANOVA.

Atxn1 RNA following a single bolus i.c.v. injection was significant throughout most of the brain regions examined and persisted in most regions for 18 weeks after i.c.v. injection. Whole cerebellum was the one region examined where Atxn1 RNA reduction failed to reach statistical significance (Figure 1A). Supplemental Figure 2 presents analyses of ATXN1[2Q] and ATXN1[66Q] protein levels in 4 brain regions at 2, 12, and 18 weeks after ASO administration. Across all regions and times after i.c.v. injection ATXN1[2Q] and ATXN1[66Q] protein levels responded similarly to ASO administration. In the cerebral cortex and cerebellum, ATXN1[2Q] and ATXN1[66Q] levels were significantly reduced by the ASO at 12 weeks after injection yet were back to untreated levels at 18 weeks after i.c.v. injection (Supplemental Figure 2, A and D). In the pons, ATXN1[2Q] and ATXN1[66Q] levels remained significantly lower than in vehicle-treated mice up to 18 weeks after injection (Supplemental Figure 2C). Finally, in the medulla, there was a decrease in ATXN1[2Q] and ATXN1[66Q] protein levels at 2 and 12 weeks after i.c.v. injection that appeared to return to untreated levels at 18 weeks after injection. However, variability of results, particularly in ATX$\mathrm{N} 1[66 \mathrm{Q}]$ from vehicle-treated mice at 18 weeks, precluded the medulla results from reaching statistical significance using 2-way ANOVA (Supplemental Figure 2B).

i.c.v. injection of Atxn1 ASO into Atxn $1^{154 Q / 20}$ mice mitigates motor deficits and prolongs survival. To examine effectiveness of the Atxn1-targeting ASO in reversing SCA1-like symptoms, we utilized Atxn154Q/20 mice that develop two key features of SCA1, notably ataxia and premature death. First, the ability of 
A

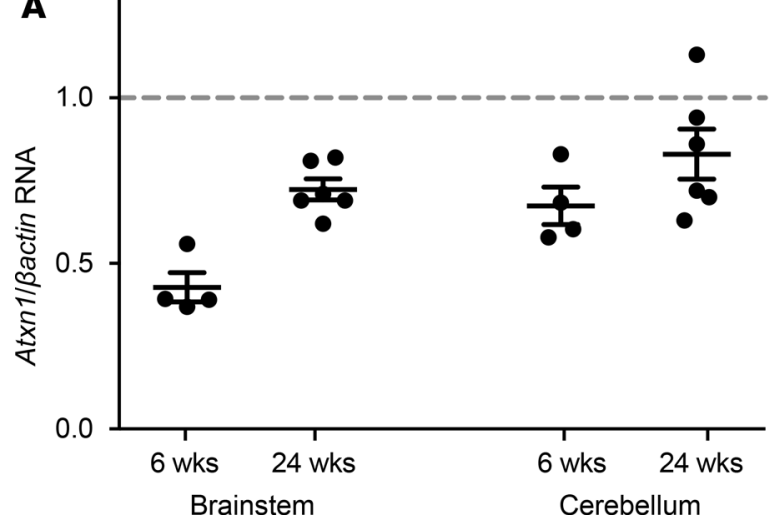

B

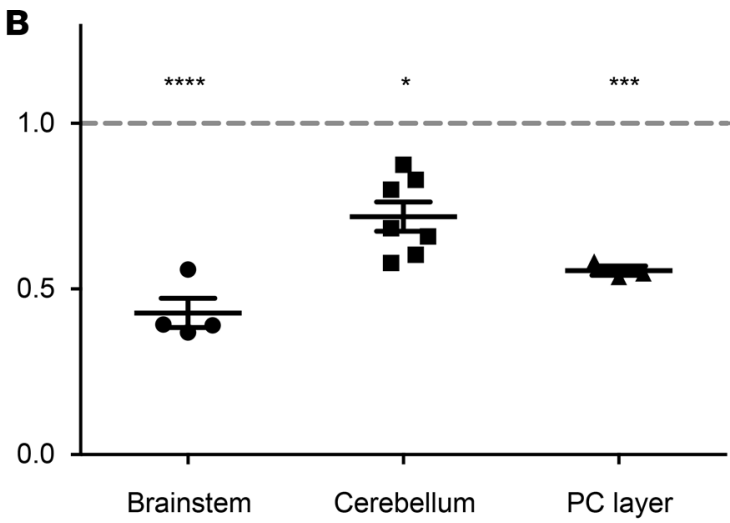

Figure 2. Reduction of Atxn1 in the brainstems, cerebella, and Purkinje cells of Atxn1 ${ }^{1540 / 20}$ mice. (A) RNA from Atxn $1^{1540 / 20}$ mice analyzed by qPCR at 6 and 24 weeks after i.c.v. injection of the ATXN1 AS0353. qPCR data are presented as the average \pm SEM; 6 -week brainstem and cerebellum $(n=6)$. (B) Atxn1 RNA brainstems, cerebella, and dissected Purkinje cells (PC layer) from Atxn1540/20 mice receiving a bolus i.c.v. injection of ASO353 (500 $\mu \mathrm{g}$ ) at 16 weeks, with analysis at 18 weeks; brainstem $(n=4)$, cerebellum $(n=7)$, Purkinje cell layer $(n=3)$. qPCR data are presented as mean \pm SEM. ${ }^{*} P<0.05$, ${ }^{* *} P<0.001,{ }^{* * *} P<0.0001$, by $t$ test.

ASO353 to reduce levels of Atxn1 RNA was examined following a bolus i.c.v. injection into the right lateral ventricle of $A t x n 1^{1540 / 20}$ mice ( $500 \mu \mathrm{g}$ in $10 \mu \mathrm{l}$ at $25 \mathrm{nl} / \mathrm{s}$ ) at 5 weeks of age. Reductions in total mouse Atxn 1 RNA were achieved in the brainstems and cerebella of $\operatorname{Atxn} 1^{154 Q / 20}$ mice at 6 and 24 weeks after a bolus i.c.v. injection of ASO353 (Figure 2A). As seen in Atxn $1^{660 / 2 O}$ mice, the degree of Atxn1 RNA reduction in $\operatorname{Atxn} 1^{1540 / 20}$ mice was greater in extracts from the brainstems compared with those from the cerebella. While Atxn1 was expressed in both Purkinje cell and granule cell layers (Supplemental Figure 3C), compared with granule cells, Purkinje cells more readily took up ASOs (Supplemental Figure 1C and ref 27). To more accurately evaluate the extent of ASO-mediated reduction of Atxn1 RNA in Purkinje cells, a prominent cellular target of SCA1 (2, 29), ASO353 was administered to Atxn1 ${ }^{1540 / 2 O}$ mice at 16 weeks of age. Atxn1 RNA levels were determined 2 weeks later in brainstem and cerebellar extracts as well as extracts prepared from the laser-dissected Purkinje cell layer (Supplemental Figure 3, A and B). ASO-mediated reduction of Atxn1 RNA in the dissected Purkinje cell layer relative to that in vehicle-injected animals was greater than that detected in whole cerebellar extracts, similar to the level of reduction detected in brainstem extracts (Figure 2B).

The ability of Atxn1 ASO353 to effect motor performance was examined by giving Atxn $1^{1540 / 20}$ mice a bolus i.c.v. injection ( $500 \mu \mathrm{g}$ in $10 \mu \mathrm{l}$ at $25 \mathrm{nl} / \mathrm{s})$ at $8-9$ weeks of age. This age was selected as an early midstage of disease, when mice have a motor deficit on the rotarod and are beginning to manifest motor deficits in home cage motor behavior (24). Mice were followed by monitoring their motor performance on the balance beam at 28 weeks of age and on the accelerating rotarod at various ages out to 31 weeks (Supplemental Figure 4A). At all ages examined, ASO353-injected Atxn $1^{1540 / 20}$ mice showed no improvement on the rotarod compared with Atxn $1^{154 / 2 O}$ mice injected with vehicle (Supplemental Figure 4A). At 28 weeks, balance beam performance of ASO-treated Atxn $1^{154 / 20}$ mice was significantly better than the performance of Atxn $1^{154 / 20}$ mice injected with vehicle. Performance of ASO-treated Atxn $1^{1540 / 20}$ mice was equal to that of vehicle-injected WT/C57BL/6 mice (Supplemental Figure 4B).

Next, the effects of administering ASO353 at a very early stage of disease (5 weeks of age) were examined when motor deficits were apparent on the accelerating rotarod, but not through observation of home cage behavior, and prior to Atxn $1^{1540 / 2 O}$ mice showing the failure to gain weight phenotype (24). Mice were assessed for motor performance using the accelerating rotarod and the balance beam at baseline at 4-5 weeks of age, just prior to ASO353 injection (Figure 3A). At 18 weeks (13 weeks after ASO injection), ASO353-injected mice showed significantly improved performance on the rotarod compared with that of vehicle-injected Atxn $1^{154 / 20}$ mice by 21 weeks of age (Figure 3A). Atxn $1^{154 / 20}$ ASO353-injected mice also showed improved motor performance on the balance beam at 15 and 26 weeks of age (Figure 3B). Rotarod performance of ASO353-injected Atxn 1540/20 mice remained significantly improved over that of vehicle-injected Atxn $1^{154 Q / 20}$ mice out to the last assessment at 27 weeks of age (22 weeks after i.c.v. injection). At this time point, rotarod assessments were ceased as vehicle-injected $\operatorname{Atxn} 1^{1540 / 20}$ mice began to die. 


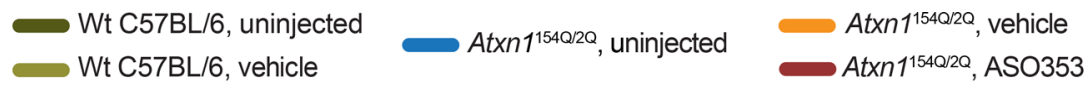

A

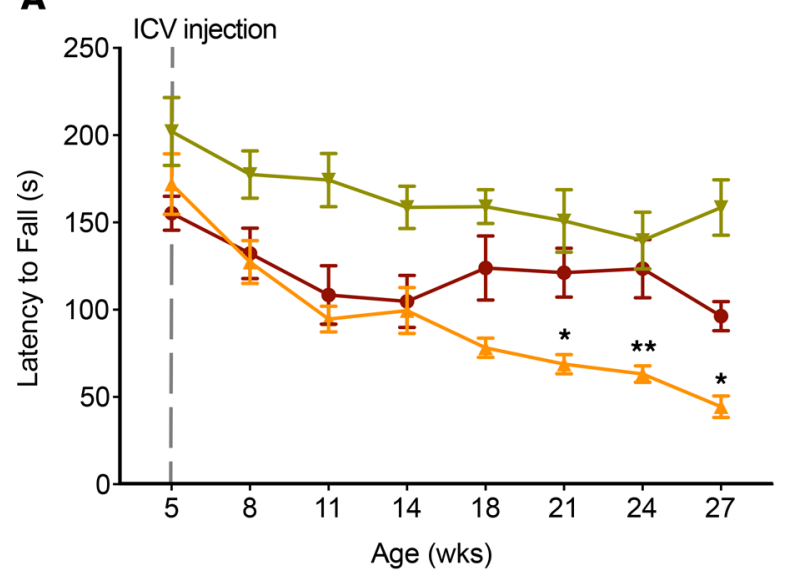

C

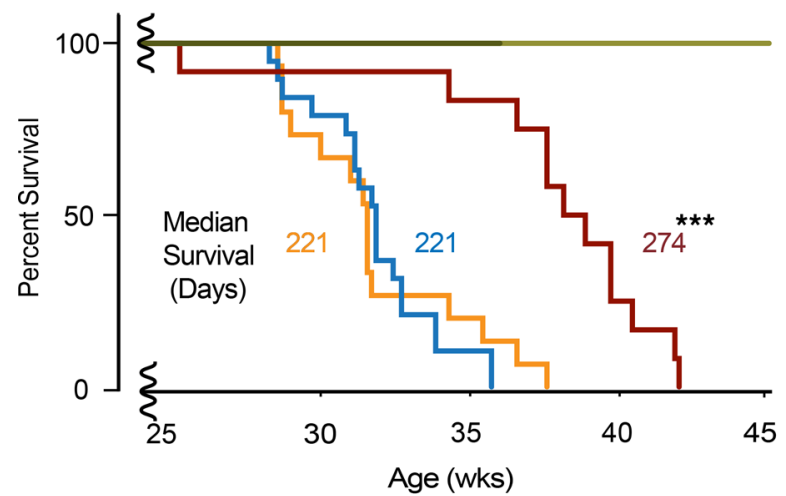

B

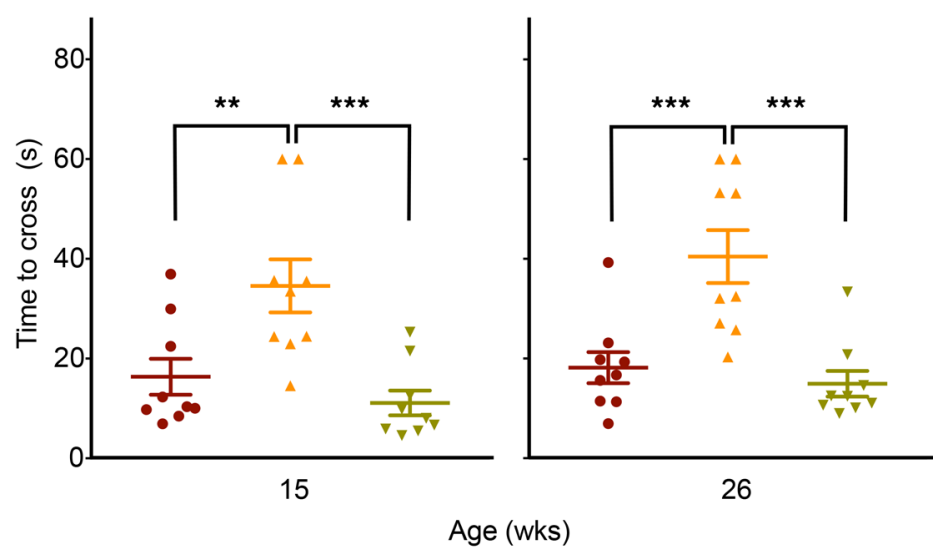

D

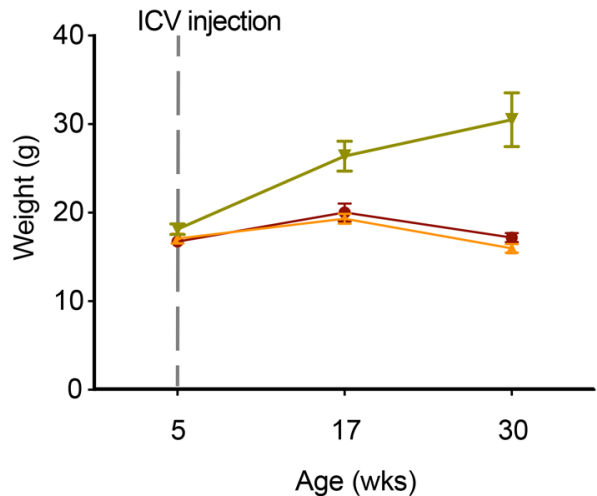

Figure 3. Recovery of SCA1-like phenotypes in Atxn1 ${ }^{1540 / 20}$ mice receiving AS0353 by i.c.v. injection at $\mathbf{5}$ weeks of age. Mice were administered ASO353 or vehicle (saline) by bolus i.c.v. injection at 5 weeks. (A) Motor performance on a rotarod between ages 5 weeks and 27 weeks. Data are presented as the mean \pm SEM. ${ }^{* *} P<0.01,{ }^{* *} P<0.001,1$-way ANOVA. (B) Motor performance on a balance beam at ages 15 weeks and 26 weeks. Data are presented as the mean \pm SEM. ${ }^{* *} P<0.01,{ }^{* *} P<0.001,1$-way ANOVA. (C) Survival, plotted as Kaplan-Meyer curves, for mice receiving a bolus i.c.v. injection of $500 \mu \mathrm{g}$ AS0353 or vehicle (saline) at 5 weeks. ${ }^{* * *} P=0.0001$, log-rank Mantel-Cox test; $P=0.0005$, Gehan-Breslow-Wilcoxon test. (D) i.c.v. injection of ASO353 fails to improve the failure-to-gain weight phenotype in Atxn1540/20 mice; WT C57BL/6 uninjected ( $n=23)$, WT C57BL/6 vehicle ( $n=16)$, Atxn1540/20 uninjected $(n=19)$, Atxn $1^{1540 / 20}$ vehicle $(n=15)$, and Atxn1 ${ }^{1540 / 20}$ ASO353 $(n=12)$.

The capability of a single Atxn1 ASO353 injection at 5 weeks of age to prolong survival of ASO353 Atxn $1^{1540 / 20}$ mice was evaluated by combining the group of mice injected for the motor performance assessment with a second cohort of mice injected solely to monitor survival. As shown in Figure 3C, an i.c.v. injection of Atxn1 ASO353 resulted in a highly significant prolongation of the survival of $\operatorname{Atxn} 1^{154 / 20}$ mice. Median survival was extended by almost 7 weeks over vehicle and uninjected Atxn $1^{1544 / 20}$ mice $(P=0.0001$ by the Mantel-Cox log-rank test and $P=0.0005$ using the Gehan-Breslow-Wilcoxon test).

Unlike the positive effects of a single ASO353 i.c.v. injection at 5 weeks on motor performance and life span of Atxn $1^{1540 / 20}$ mice, i.c.v. injected Atxn1 ASO353 was unable to alleviate the failure of Atxn $1^{1540 / 20}$ mice to gain weight with age (Figure 3D). Since ASOs cross the blood brain barrier poorly (30), these results might be evidence of either peripheral pathology or pathology in a brain region not as accessible to the ASO.

Differential gene expression and gene coexpression networks - an overview of Atxn $1^{1540 / 20}$ disease in pons, cerebellum, and medulla. The ability of Atxn1 ASO353 to mitigate SCA1-like symptoms of motor performance deficits (ataxia) and premature lethality in Atxn $1^{1540 / 2 Q}$ mice coupled with the physiologically accurate spatial and temporal expression of ATXN1[154Q] provides an experimental platform with which to compare disease-associated molecular pathways in different regions of the CNS affected by SCA1 pathology. Histological $(2,29)$ and MRI studies $(31)$ of SCA1 patients indicate that the greatest neuronal loss occurs in the cerebellum and brainstem (pons and medulla). In addition, within the hypoglossal nucleus of Atxn1 ${ }^{1540 / 20}$ 
mice, included within the medulla portion of the dissection (Supplemental Figure 5A), there are signs of neuronal pathology and astrocytosis by 6 months of age (4). Thus, we focused our analyses on these regions.

To compare molecular aspects of SCA1-like disease in the cerebella and brainstems of Atxn $1^{154 Q / 2 Q}$ animals given a bolus ASO353 i.c.v. injection at 5 weeks, we obtained RNA-seq data sets on polyA ${ }^{+}$RNA from vehicle- and Atxn1 ASO353-treated Atxn $1^{154 Q / 20}$ cerebella at 18 weeks of age. Atxn $1^{154 Q / 2 Q}$ pons and medulla, including the inferior olive and hypoglossal nuclei, were also analyzed at both 18 and 28 weeks of age (see Supplemental Table 1 for summary of RNA-seq analyses). As shown in Figure 3, A and C, these ages correspond to time points at the initiation of improvement in motor performance on the rotarod (18 weeks) and just prior to extension of survival (28 weeks) in Atxn1 ASO353-treated Atxn $1^{1540 / 20}$ mice.

Consistent with previous RNA-seq analysis of Pcp2-ATXN1[82Q] cerebella (25), there was a sizeable number of differentially expressed (DE) genes in Atxn $1^{154 Q / 2 Q}$ cerebella compared with WT cerebella at 18 weeks of age, with a majority being downregulated (Figure 4A and Supplemental Table 2). In contrast, essentially no DE genes were detected in the pontes and medullae of 18-week-old Atxn $1^{1540 / 20}$ mice. By 28 weeks of age, a robust number of DE genes were found in Atxn $1^{154 Q / 2 Q}$ pontes and medullae, with the number of downregulated DE genes being slightly more than the number of upregulated DE genes (Figure 4A and Supplemental Table 2). Therefore, disease in Atxn $1^{1540 / 20}$ mice begins considerably later in the pons and medulla than in the cerebellum, as by RNA-seq-based assessment. Supplemental Figure 5B shows that developmental onset of Atxn1 expression was not delayed in the pons and medulla compared with the cerebellum. Thus, delay in onset of disease in the pons and medulla, when compared with onset of disease in the cerebellum, is unlikely to be due to a simple delay in onset of Atxn1 expression in the pons and medulla. Importantly, the numbers of DE genes were substantially reduced in all 3 brain regions examined in Atxn $1^{154 Q / 2 Q}$ mice treated with Atxn1 ASO353 at 5 weeks of age (Figure 4B). The reduction in DE genes in ASO353-treated Atxn1 $1^{154 \mathrm{Q} / 20}$ mice ranged from a high of $92 \%(1,430$ to $109 \mathrm{DE}$ genes) in the medulla at 28 weeks to a low of $79 \%$ (2,947 to $613 \mathrm{DE}$ genes) in the pons at 28 weeks.

Gene set enrichment analysis (GSEA) allows a comparison of the distribution of a defined set of genes (32), e.g., the cerebellar Magenta gene coexpression module, within a ranked ordered list of genes generated from a RNA-seq analysis of a group of samples, i.e., DE genes from 18 -week-old vehicle-treated Atxn1 ${ }^{154 Q / 2 O}$ mice compared with WT mice ranked by direction and degree of differential expression. The more clustered the distribution of the Magenta gene set is toward one end or the other of the list of the Atxn $1^{1540 / 20}$ cerebellar $\mathrm{DE}$ genes (skewed toward the top or bottom of the ordered list), the more the defined gene set correlates with the phenotypic class represented by the gene list, i.e., disease. Figure 4C shows the result of a GSEA of the Magenta module gene set (Magenta-GSEA) compared with the list of cerebellar DE genes from 18-weekold WT vehicle versus treated Atxn $1^{154 Q / 2 Q}$ mice. Distribution of genes within the Magenta module is highly skewed (NES $=-11.43, P<0.0001)$ toward the end of the gene list containing genes with lowest expression in the cerebella of 18 -week-old $\operatorname{Atxn} 1^{154 Q / 20}$ vehicle-treated mice versus vehicle-treated WT mice. While the medulla Magenta-GSEA also showed some skewing of Magenta genes toward the end of the 28-week-old Atxn $1^{154 Q / 2 Q} \mathrm{DE}$ gene list containing genes with lowest level of expression (NES $=-5.60, P<0.0001$ ), the enrichment score curve for the medulla Magenta-GSEA analysis was considerably flatter than the enrichment score curve for the cerebellar Magenta-GSEA (Figure 4D). Thus, these Magenta-GSEAs support the concept that cerebellar disease in Pcp2-ATXN1[82Q] mice correlates highly with cerebellar disease in Atxn154Q/2Q mice and to a lesser degree with disease in the medulla of Atxn $1^{154 / 20}$ mice.

Brainstem disease-associated transcriptome profile in Atxn $1^{1540 / 20}$ mice is distinct from the cerebellar diseaseassociated transcriptome profile. Weighted gene coexpression network analysis (WGCNA), a reproducible and effective approach to link coexpressed gene modules with phenotypic traits $(33,34)$, was recently used to generate the Magenta coexpression module and correlate it to Purkinje cell disease progression in ATXN1-transgenic mice (25). WGCNA applied to the medulla RNA-seq data set at 28 weeks of age revealed a module of 1,510 genes, designated the Brown module, that is highly associated with disease ( $t$ test, $P=10^{-8}$ ) in $A t x n 1^{154 Q / 2 Q}$ mice. To further assess the Brown medulla gene network connection to disease, a transcriptional heatmap analysis of the Brown gene module was generated (Figure 5). This analysis showed that in the medulla at 28 weeks ASO-treated Atxn $1^{154 Q / 2 Q}$ mice clustered with the WT group (Figure $5 \mathrm{~A})$. In contrast, a similar analysis of the Brown module in the cerebellum data set at 18 weeks revealed considerably less evidence of clustering of ASO-treated Atxn $1^{1540 / 20}$ mice with the WT mice (Figure 5B). We interpret the results of these transcriptional heatmap analyses as support for the concept that the Brown gene network has a greater role in disease in the medullae than in the cerebella of Atxn $1^{154 Q / 20}$ mice. 
A

WT veh vs Atxn $1^{154 Q / 2 Q}$ veh
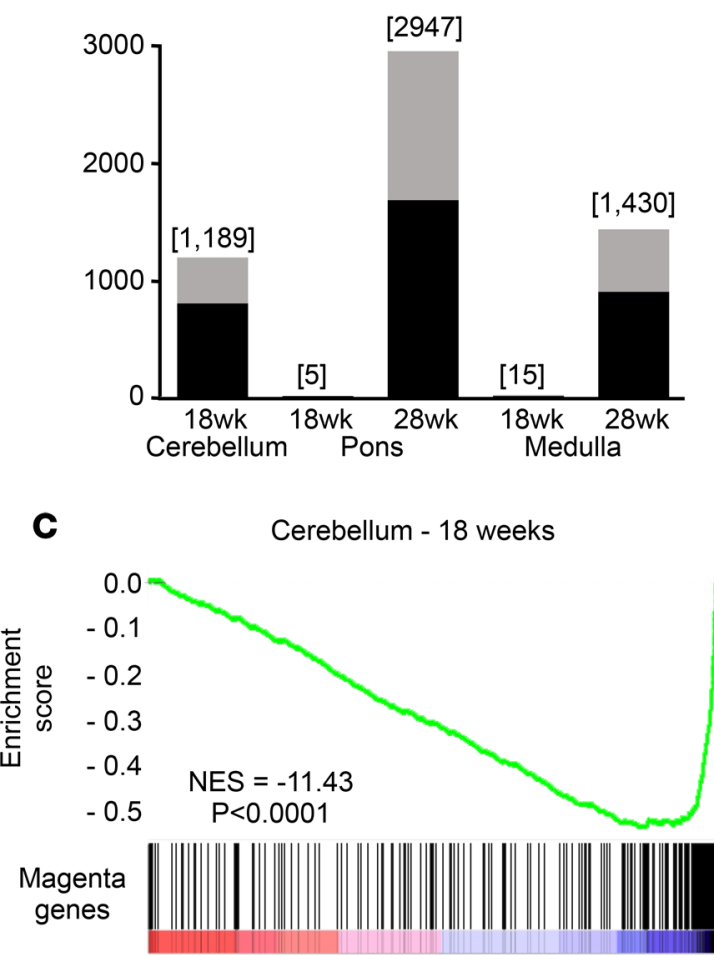

Gene list rank

WT veh vs Atxn 1154Q/2Q veh
B

Atxn1 $1^{154 Q / 2 Q}$ veh vs Atxn1 ${ }^{154 Q / 2 Q}$ ASO353

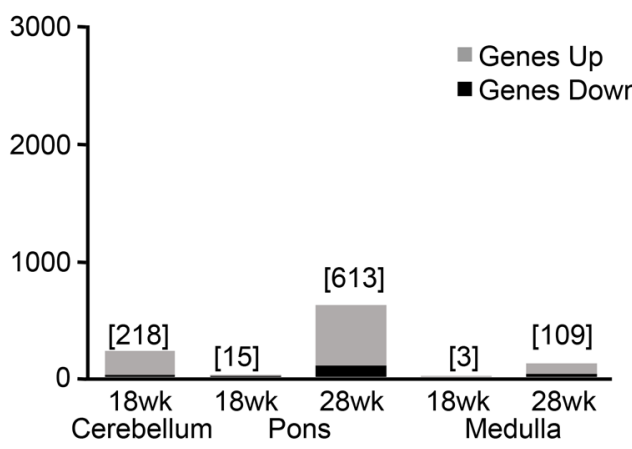

D

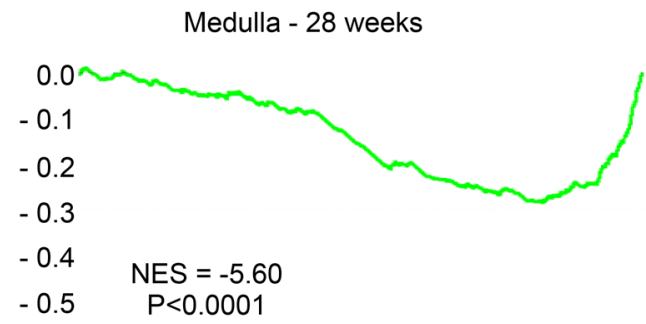

Magenta

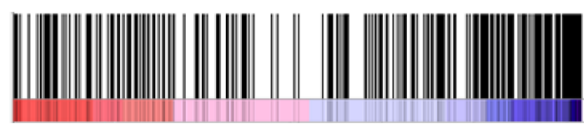

Gene list rank

WT veh vs Atxn $1^{154 \mathrm{Q} / 2 \mathrm{Q}}$ veh

Figure 4. Differentially expressed genes in the cerebella, pontes, and medullae of Atxn1 ${ }^{1540 / 20}$ mice receiving vehicle or AS0353 by i.c.v. (A) Genes differentially expressed in vehicle-treated WT versus vehicle-treated Atxn 1540/20 mice from the cerebellum at 18 weeks, the pons at 18 and 28 weeks, and the medulla at 18 and 28 weeks. The black portion of the bars depicts the number of genes downregulated and the gray portion of the bars depicts the number of genes upregulated in vehicle-treated Atxn $1^{1540 / 20}$ mice. (B) Genes differentially expressed in vehicle-treated Atxn $1^{1540 / 20}$ versus ASO353-treated Atxn $1^{1540 / 20}$ mice from the cerebellum at 18 weeks, the pons at 18 and 28 weeks, and the medulla at 18 and 28 weeks. The black portion of the bars depicts the number of genes downregulated and the gray portion of the bars depicts the number of genes upregulated in ASO353-treated Atxn ${ }^{1540 / 20}$ mice. In $\mathbf{A}$ and $\mathbf{B}$, numbers above bars indicate the number genes with differential expression. (C and D) CSEA of the Magenta module compared with differentially expressed gene lists from the cerebella of 18 -week-old and medullae of 28 -week-old Atxn $1^{1540 / 20}$ mice, respectively. Significance was determined by the observed enrichment score compared to a set of null scores generated by permuting the gene ranked order 10,000 times.

To query further the similarity between the medulla and cerebellar disease-associated WGCNA gene networks, we generated a connectivity diagram identifying the hub genes, i.e., the core, most highly connected genes in the module, for the medulla Brown module. When compared with the cerebellar Magenta module (25), only 1 of the top 10 Magenta hub genes was found in the Brown module, and only 1 of the top 10 Brown hub genes was found in the Magenta module (Supplemental Table 3). Moreover, of the top 100 Magenta genes, only 16 are found in the Brown module, with the highest ranking of these 16 genes ranked 233rd in the Brown module. Conversely, of the top 100 Brown module genes, only 6 of these are in the Magenta module, with the highest ranking of these genes ranked 166th in the Magenta module.

Next, we determined the overlap in genes between the medulla Brown module and the previously generated cerebellar Magenta module as a means of comparing the disease process in these two regions of the CNS. To accurately assess the overlap between the Magenta cerebellum module and the Brown medulla module, we first restricted the comparison to the set of all genes that were detected in both WGCNA analyses (i.e., those genes that met minimum expression thresholds in both tissue types). There were 11,336 genes in common between the WGCNA analyses. Of the 342 Magenta module genes and 1,510 medulla Brown module genes, respectively, 333 and 1,053 were in the set of 11,336 genes shared by both analyses. Of these two reduced module lists, there was a significant overlap $(P<4.607 \mathrm{E}-09)$ of 65 genes (Figure 6). When Ingenuity Pathway Analysis (IPA) was performed on the Magenta and Brown modules, the top most significant canonical pathways for each module were quite distinct (Figure 6). As shown previously (25), the Magenta IPA canonical pathways were enriched for signaling pathways. The Brown IPA pathways, on 
A

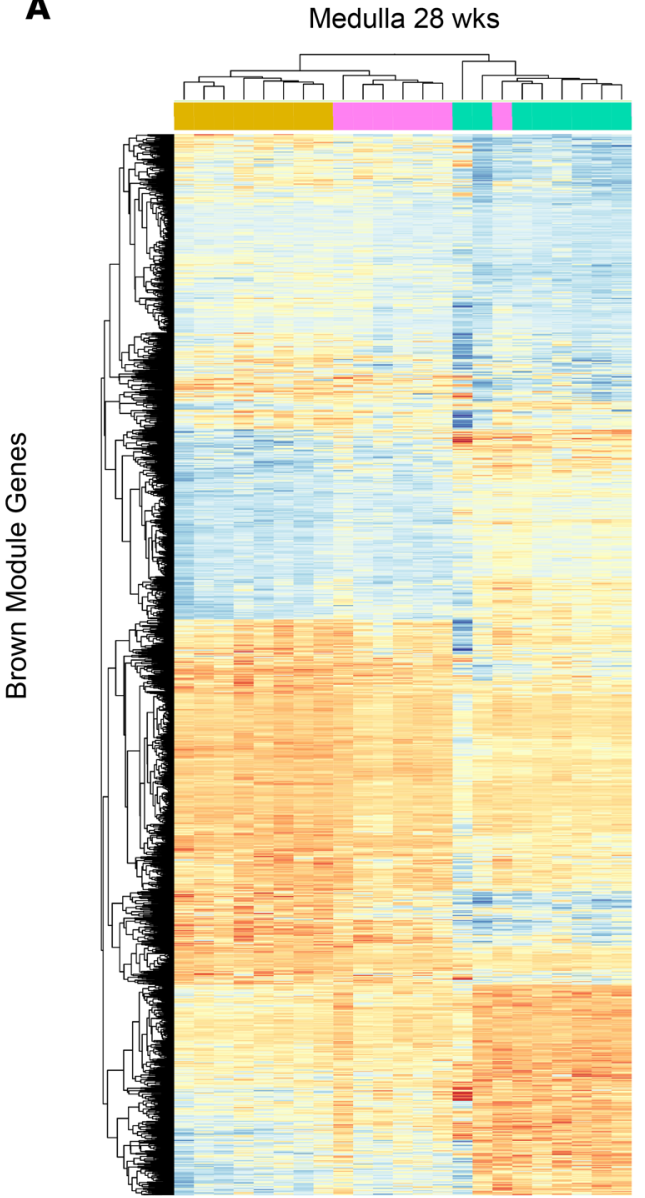

B

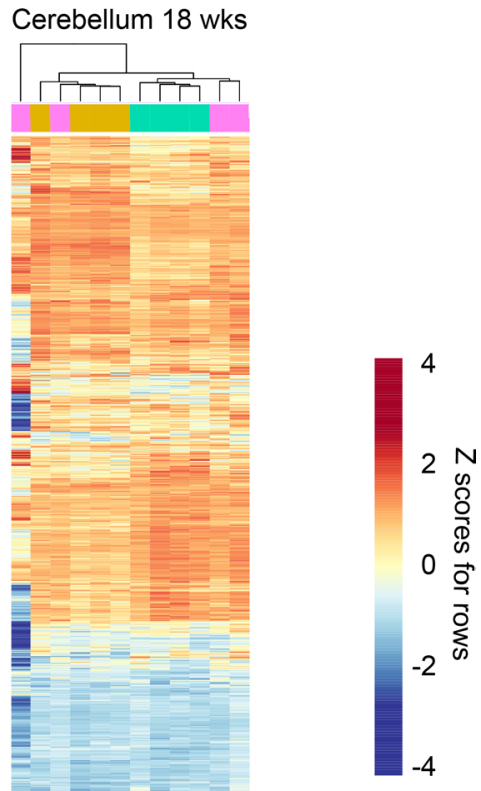

Figure 5. Medulla Brown WCCNA module is associated with disease in Atxn1540/20 mice. (A) Transcriptional heatmap of Brown module genes for the medulla of vehicle- and ASO353-treated Atxn1540/20 mice and vehicle-treated WT mice. (B) Transcriptional heatmap of Brown module genes for the cerebella of vehicle and AS0353-treated Atxn 1540/20 mice and vehicle-treated WT mice; ASO-treated Atxn1540/20 mice $(n=7)$, vehicle-treated Atxn 1540/20 mice $(n=7)$, and vehicle-treated WT mice $(n=8)$.

the other hand, included several metabolic/biosynthetic pathways and none of the Magenta signaling pathways. While the set of 65 overlapping genes had no significant IPA pathways after Benjamini-Hochberg correction for multiple testing, it is interesting that cyclin-dependent kinase- 5 (CDK5) signaling was the top uncorrected pathway (Figure 6), since Cdk5 regulates several fundamental processes thought to have a role in neurodegeneration, including neuronal survival, neurite, and axonal outgrowth (35).

Finally, the cerebellar Magenta WGCNA module is significantly enriched for genes having a CIC-binding site within their upstream region (25). This is an important aspect of the Magenta module that further links this module to pathogenesis in the cerebellum. Formation of the ATXN1/CIC complex is critical for polyglutamine-expanded ATXN1-mediated toxicity in Purkinje cells (36). In contrast, the upstream regions of the 1,510 genes in the medulla Brown module were far less enriched for the transcriptional regulator CIC-binding sites than the Magenta genes (Table 1). Taken together, we concluded that the cerebellar Magenta and medulla Brown disease-associated WGCNA modules represent distinct gene coexpression networks, suggesting that the pathogenic pathways differ considerably between the cerebellum and medulla.

Pons WGCNA in Atxn1 ${ }^{154 / 20}$ mice. Application of WGCNA to the pons RNA-seq data set yielded 4 WGCNA modules with a significant correlation with disease, with disease-associated significances of $P=$ $10^{-2}$ to $10^{-3}$; these were much lower than the level of significance for disease association seen with either the cerebellar Magenta module $\left(P=10^{-9}\right.$; ref 24$)$ or the Brown medulla module $\left(P=10^{-8}\right.$; this study). 


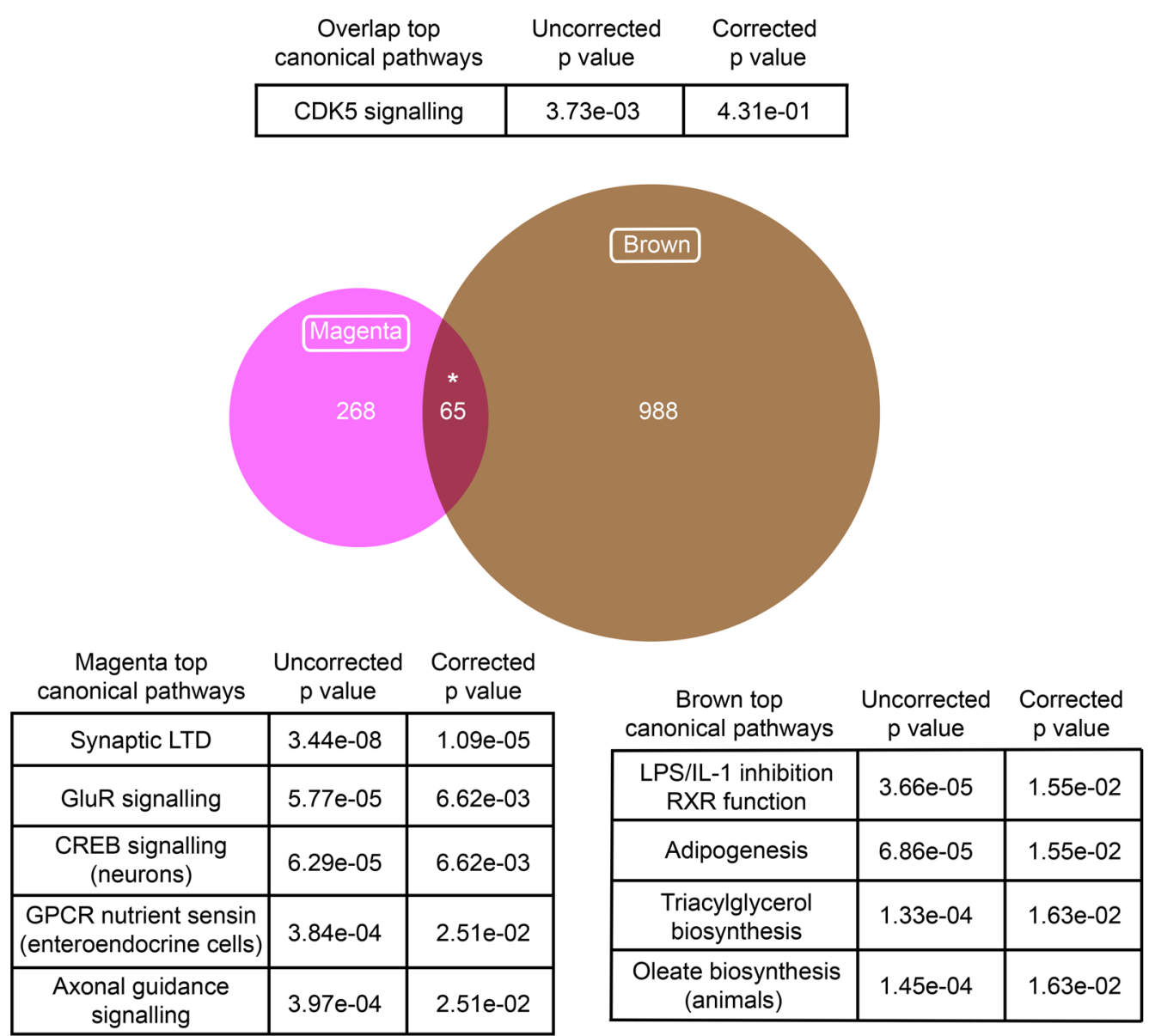

Figure 6. Gene overlap between cerebellar Magenta and medulla Brown WGCNA modules. Venn diagram depicting gene overlap between the cerebellar Magenta and medulla Brown WGCNA modules for genes in common between both analyses. The overlap of 65 genes is significant at $P<4.61 \mathrm{E}-05$. The top IPA canonical pathways for each module and the overlapping genes are listed. While no canonical pathways were significant for the overlapping gene set with Benjamini-Hochberg correction for multiple tests, the top canonical pathway in the uncorrected analysis is shown.

Thus, Atxn1[154Q]-mediated disease in the mouse pons did not manifest with a distinct set of coexpressed genes robustly linked to disease. A likely explanation for the absence of a disease-associated coexpressed gene network in the mouse pons is that the relative amount of the area typically affected in SCA1, the basal pons, is only a small fraction of what is present in the human pons. Pontine pathology, when present in human SCA1, typically involves the basal pontine nuclei and their projections via the transverse fibers to the cerebellar cortex (29). In the human brain, the majority of the pons (up to $75 \%-80 \%$ ) is composed of the basal pontine region (Supplemental Figure 6A). The cross-sectional area of the basal pons in the mouse is only $10 \%-15 \%$ of the total pontine region (Supplemental Figure $6 \mathrm{~B}$ ), most of which is composed of tegmental structures. In mice, the relative amount of the area of pons that would be typically affected in SCA1 is a small fraction of what is present in humans, providing a biological basis for the inability to identify a disease-associated WGCNA coexpression gene network in the pons of Atxn $1^{154 / 20}$ mice.

The inability to detect disease-associated coexpression gene networks in the pons when the pontes of $\operatorname{Atxn} 1^{1540 / 20}$ mice showed numerous alterations in gene expression that were decreased with ASO353 administration, as shown by differential gene analysis, seems contradictory. Recently, Al-Ramahi et al. performed a high-throughput functional analysis of transcriptional changes using a Drosophila HD model (37), screening 312 genes that are dysregulated both in patients and mouse models of HD. In addition to finding transcriptional changes that are drivers of disease or compensatory changes, they showed that a majority of alterations in gene expression, 230 of the 312 genes, had no consequence at all. Thus, we suggest that even a greater proportion of the numerous transcriptional changes in pontes 
Table 1. Enrichment of genes with a CIC-binding motif in the Magenta and Brown WGCNA modules

\begin{tabular}{|c|c|c|c|c|c|c|c|}
\hline CIC-binding motif & Module & & & CIC-binding motif & Module & & \\
\hline Genes motif absent & 190 & 278 & & Genes motif absent & 1,160 & 1,232 & \\
\hline TGAATGGA & & & & TGAATGGA & & & \\
\hline Genes motif present (\%) & $180(53.1 \%)$ & 100 & 5.85E-10 & Genes motif present (\%) & $272(18.0 \%)$ & 274 & 0.962 \\
\hline
\end{tabular}

of Atxn $1^{154 Q / 20}$ mice, a region of the mouse CNS that lacks the anatomical portion affected by SCA1, likely fall into the nonconsequential category described by Al-Ramahi et al. (37).

Neurochemical response to ASO treatment in Atxn $1^{154 Q / 2 Q}$ mice. Previously, we demonstrated that MRS accurately reflected recovery of cerebellar pathology in a conditional transgenic mouse model of ATXN1[82Q]-induced cerebellar disease $(10,11)$. Consequently, MRS was examined for its ability to detect ASO treatment effects on neurochemicals in the cerebella and brainstems in Atxn $1^{154 Q / 2 Q}$ mice. High-quality MR spectra (Figure 7, A and D) yielded 16 reliably quantified metabolites in both regions (Supplemental Figure 7). Of the 65 mice scanned, 2 mice were identified as having the abnormal high-glutamine phenotype, a consequence of portosystemic shunting in C57BL/6 mice that was previously described $(38,39)$. The MR spectra of these 2 mice were not included in the final analysis. Differences in cerebellar taurine (Tau, an osmolyte, neuromodulator, and neuroprotectant; ref. 40), total choline (tCho, potential marker of disturbances in membrane phospholipid metabolism), total creatine (an energy metabolism or glial marker), and glutamine (a potential glial or osmolytic marker) in Atxn $1^{154 Q / 2 Q}$ mice compared with WT mice at 18 and 28 weeks (Supplemental Figure 7, A and C) matched previously described findings in this mouse line (39). In addition, lower $N$-acetylaspartylglutamate (NAA, a neuronal marker) (41), total NAA (tNAA), and myo-inositol (Ins, potential glial or osmolytic marker) levels were detected in the cerebellum at both 18 and 28 weeks, and lower glutamate (a neurotransmitter) was detected at 28 weeks in the vehicle-treated $A t x n 1^{154 Q / 2 Q}$ group versus the WT group $(P<0.05)$. ASO treatment reversed the Ins and tCho abnormality in the cerebella of $\operatorname{Atxn} 1^{154 Q / 2 Q}$ mice at 18 weeks (Figure 7B), while reversal was partial at 28 weeks $(P<0.05$ at both ages and for both metabolites) (Figure 7C). A subset of the cerebellar neurochemical abnormalities was also detected in the brainstems of Atxn $1^{154 Q / 2 Q}$ mice versus WT mice, including lower NAA, tNAA, tCho, Ins, and Tau (Supplemental Figure 7, B and D). Of these, lower Ins levels were fully reversed with ASO treatment at 18 weeks (Figure 7E) and partially reversed at 28 weeks (Figure 7F).

\section{Discussion}

In this study, we found that a single i.c.v. bolus injection of an ASO-targeting mouse Atxn1 RNA resulted in significant reduction in motor deficits as well as prolonged survival of Atxn $1^{154 Q / 2 O}$ mice. Injection of ASO353 at 5 weeks of age resulted in substantial improvement of motor performance on the balance beam and accelerating rotarod, while an injection at 8-9 weeks of age yielded an improvement on the balance beam, without improved performance on the accelerating rotarod. These latter results are consistent with the conditional model of ATXN1[82Q]-induced Purkinje cell disease, where the degree of reduction in motor deficits was age dependent (9). The Atxn1 RNA-targeting ASO353 results in Atxn1 ${ }^{154 Q / 2 Q}$ mice add to the growing potential of ASO therapy for dominantly inherited diseases (18), including other polyglutamine diseases, such as HD (28), SCA2, and SCA3 (20, 21).

Analyses of transcriptomic data from the ASO353-treated Atxn $1^{1540 / 20}$ mice supports three key conclusions. First, in contrast to the WGCNA results from RNA-seq data from the cerebellum and medulla (see below), the inability to detect a disease-associated WGCNA coexpression gene network in the pontes of Atxn $1^{154 Q / 2 Q}$ mice reinforces the usefulness of the WGCNA strategy as a means of identifying gene expression changes associated with a SCA1-like phenotype.

Second, we found that the WGCN Magenta module gene set derived from cerebellar RNA-seq data from mice, in which expression of ATXN1[82Q] is restricted to Purkinje cells, significantly overlapped with 

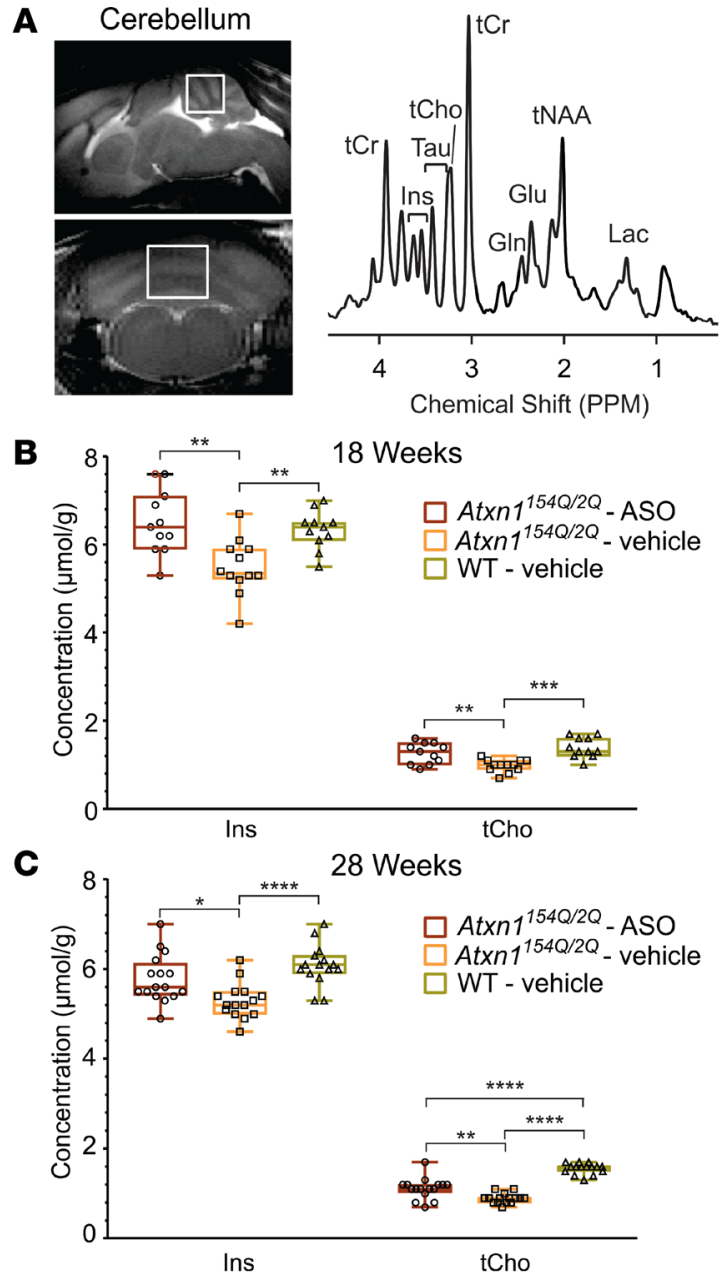
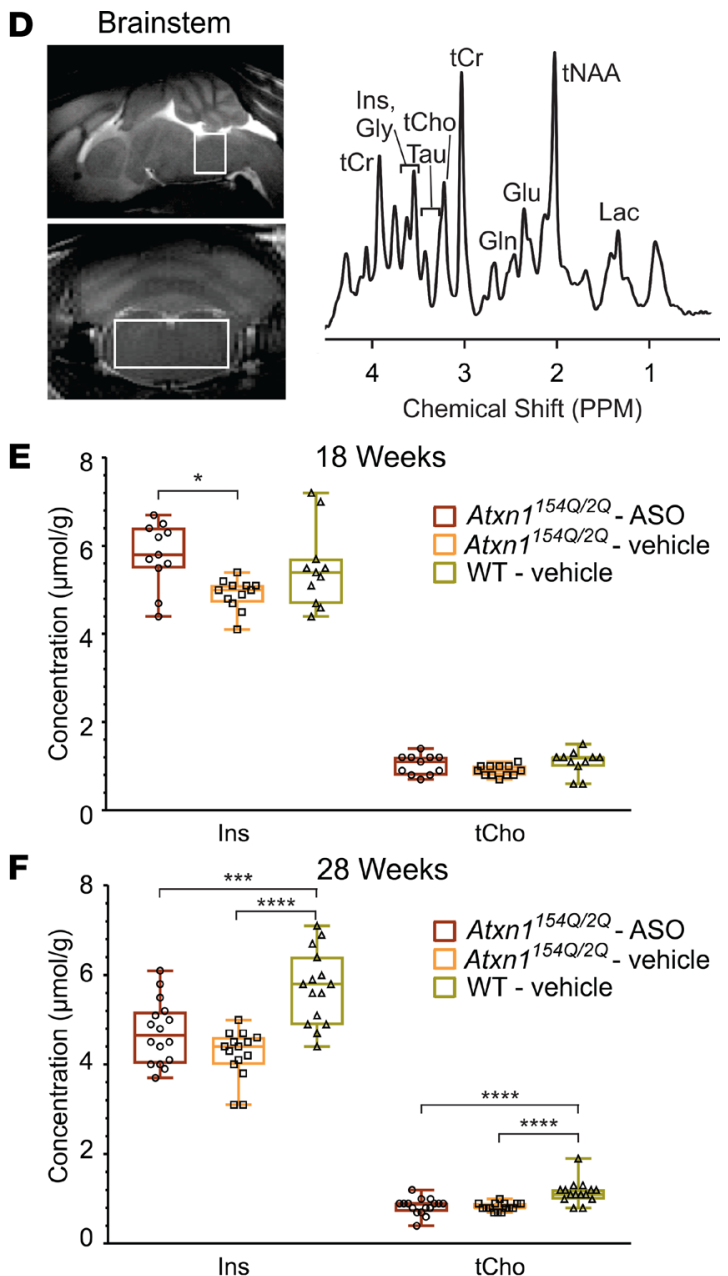

Figure 7. ASO treatment effects on neurochemicals in the cerebella and brainstems in Atxn ${ }^{1540 / 20}$ mice. (A and D) Voxel placement and spectral quality in the cerebellum and brainstem. $T_{2}$-weighted images and localized proton MR spectra (LASER, TE = $15 \mathrm{~ms}, T R=5 \mathrm{~s}$ ) are shown for an ASO353-treated Atxn1 1540/20 mouse. (B, C, E, and F) Myo-inositol (Ins) and total choline (tCho) concentrations in the cerebella (B and C) and brainstems (E and F) of AS0353-treated Atxn 1540/20 mice, vehicle-treated Atxn 1540/20 mice, and vehicle-treated WT mice at 18 and 28 weeks of age. Sample sizes in B and $\mathbf{E}$ are as follows: ASO-treated Atxn1540/20 mice $(n=11)$, vehicle-treated Atxn1540/20 mice $(n=12)$, and WT mice $(n=11)$. Sample sizes in $\mathbf{C}$ and $\mathbf{F}$ are as follows: ASO-treated Atxn1540/20 mice $(n=16)$, vehicle-treated Atxn1540/20 mice $(n=15)$, and WT mice $(n=15)$. In the box-and-whisker plots, the bounds of the boxes represent 25 th to 75 th percentiles, the line in the box is the median, and the whiskers extend to the minimum and maximum values. Group means for neurochemical concentrations were compared with 1-way ANOVA, and $P$ values were corrected for multiple comparisons using the Tukey Method. ${ }^{*} P<0.05 ;{ }^{* *} P<0.01 ;{ }^{* *} P<0.001 ;{ }^{* * *} P<0.0001$

cerebellar DE genes that were corrected to WT expression in the cerebella of ASO353-treated Atxn1 1540/20 mice. In addition, the GSEA of the Magenta module comparing the list of cerebellar DE genes from cerebella of 18 week-old WT vehicle-treated mice with $A t x n 1^{1540 / 2 Q}$ vehicle-treated mice indicated that the Magenta module highly correlates with cerebellar disease in Atxn $1^{1540 / 20}$ mice. Thus, we conclude that cerebellar disease in Atxn $1^{154 Q / 2 Q}$ mice is largely driven by effects of expanded ATXN1 on Purkinje cells.

Third, RNA-seq data from ASO353-treated Atxn $1^{154 Q / 2 Q}$ mice indicates that the disease processes in the cerebellum that underlie ataxia differ considerably from those in the medulla that presumably contribute to the shortened life span seen in SCA1. While there appears to be some overlap in the effect of expanded ATXN1 on gene expression in the cerebellum and medulla, key features of gene expression changes in the medulla support the concept that seminal differences in disease processes exist between the medulla and the cerebellum. As measured by alterations in gene expression, onset of disease is delayed considerably in the medulla compared with the cerebellum. At 18 weeks of age, Atxn $1^{154 Q / 2 O}$ mice showed no signs of altered gene expression in the medulla, while a substantial number of DE genes were detectable in the cerebella of Atxn $1^{154 Q / 2 Q}$ mice at the same age. By 28 weeks of age, DE genes were detectable in the medullae of $\operatorname{Atxn} 1^{154 Q / 2 Q}$ mice. 
As assessed by WGCNA analyses of altered gene expression in Atxn $1^{1540 / 20}$ mice, genes within the medulla Brown coexpression module have little overlap with the cerebellar Magenta module. In addition, the cerebellar Magenta module is enriched for genes whose expression is regulated by the ATXN1-interacting transcription factor CIC (25). Importantly, the ability of expanded ATXN1 to interact with CIC is critical for it to induce disease in Purkinje cells (36). In contrast to the cerebellar Magenta gene network, the medulla Brown module is only significantly enriched for genes with 1 of the 2 CIC-binding site motifs in their upstream regions and far less significantly enriched than the Magenta module. Thus, genes in the medulla Brown module are unlikely to be regulated by CIC, suggesting that the interaction of ATXN1 with CIC is not a major driver of disease in the medulla. This conclusion is consistent with the finding that, while loss of CIC function has a major effect on reducing cerebellar motor phenotypes, it has far less of an effect on extending life span in Atxn $1^{154 Q / 2 Q}$ mice (42). Future work looking at the extent to which additional ATXN1 interactors mediate neurodegeneration in the medulla will be critical for therapeutic considerations going forward.

The MRS findings were largely consistent with the analyses of transcriptomic data. First, we detected a smaller number of neurochemical abnormalities and a more limited ASO response in the brainstems of the Atxn $1^{154 Q / 2 Q}$ mice than in the cerebella at both 18 and 28 weeks. This is likely due to partial volume effects, namely the brainstem volume of interest (VOI) contained both pontine and medullary tissue (Figure 7D). Additionally, a small fraction of the pons is affected by SCA1 in the Atxn $1^{1540 / 20}$ mice, as also shown by the lack of a disease-associated WGCNA coexpression gene network in the pons. In contrast, the majority of the pons is affected in patients with SCA1. Consistently, pontine neurochemicals show strong correlations with clinical status (6) and pons volume measured by MRI is the most sensitive structural measure to change in patients with SCA1 (31). Therefore, MRS of the pons is still expected to be sensitive to treatment effects in human subjects undergoing gene-silencing therapies. Second, similar to the gene expression findings, onset of neurochemical abnormalities was delayed in the brainstem compared with the onset in the cerebellum. At 18 weeks of age, while numerous neurochemical levels were abnormal in the cerebellum (Supplemental Figure 7A), Atxn $1^{1540 / 20}$ mice showed minimal neurochemical abnormalities in the brainstem (Supplemental Figure 7B). By 28 weeks of age, a substantially larger number of neurochemical differences were detectable in the brainstems of Atxn $1^{154 Q / 2 Q}$ mice, while still fewer than those detected in the cerebella (Supplemental Figure 7, C and D). The limited and delayed pathology in the tissue covered by the brainstem VOI likely resulted in the limited neurochemical response to a single ASO injection administered at 5 weeks.

Notably, the classic markers of neuronal health, NAA and glutamate, were unaffected by ASO treatment. Instead, two neurochemicals that represent early biochemical changes prior to gross degenerative pathology $(39,43)$, namely Ins and tCho, were fully or partially rescued at 18 and 28 weeks of age following ASO treatment. Of these, increased Ins is typically considered a marker of gliosis (41). Because we detected lower rather than higher Ins levels in the Atxn $1^{154 Q / 2 Q}$ versus WT mice, this neurochemical abnormality likely reflects an osmolytic imbalance in these mice. Consequently, the increase in Ins following ASO injection is likely related to osmolytic effects of the treatment. Similarly, increased levels of tCho are typically associated with increased membrane turnover (44). Therefore, the lower tCho levels detected in Atxn $1^{1540 / 20}$ mice compared with WT more likely reflect disturbances in membrane phospholipid metabolism (45), which are likely rescued with ASO treatment.

Importantly, this work provides proof of concept for the ability of an ATXN1-targeting ASO to rescue two seminal SCA1 phenotypes: ataxia and premature lethality. In addition, the data indicate that regional differences in disease processes between the cerebellum and medulla are large enough that a therapy designed to target a specific cellular pathogenic pathway active in one region is unlikely to substantially effect disease in the other. These findings further support the therapeutic value of an approach such as ASO-mediated reduction of ATXN1 RNA, which is effective across multiple regions of the brain and the corresponding cellular pathways affected by SCA1.

\section{Methods}

Mice. All mice were housed and managed by Research Animal Resources under specific pathogen-free conditions in an Association for Assessment and Accreditation of Laboratory Animal Care Internationalapproved facility. In all experiments, equal numbers of male and female mice were used. For tissue analyses, mice were harvested via $\mathrm{CO}_{2}$ euthanasia and brains were dissected to isolate the cortex, cerebellum, pons, and brainstem. Each region was divided in half and frozen in liquid nitrogen. For i.c.v. injections, 
mice were anesthetized with an intraperitoneal ketamine/xylazine cocktail (100 mg/kg ketamine and 10 $\mathrm{mg} / \mathrm{kg}$ xylazine). Using a stereotax, the cranium was burr drilled and a Hamilton Neuros Syringe (65460$05)$ was positioned at the following coordinates: $\mathrm{AP}, 0.3 ; \mathrm{ML}, 1.0 ; \mathrm{DV},-2.7 \mathrm{~mm}$ from bregma. Injections of $10 \mu \mathrm{ASO} 618353(50 \mu \mathrm{g} / \mu \mathrm{l})$ dissolved in PBS without $\mathrm{Ca} / \mathrm{Mg}(\mathrm{Gibco}, 14190)$ or vehicle were delivered via a micro-syringe pump at $25 \mathrm{nl} / \mathrm{s}$. Immediate postoperative care included subcutaneous delivery of 250 $\mu \mathrm{l}$ saline, carprofen $(7 \mathrm{mg} / \mathrm{kg}$ ) and Buprenorphine SR (Zoopharm Pharmacy) $(2 \mathrm{mg} / \mathrm{kg}$ ).

Western blots. One half of each brain region from each mouse was homogenized using a tissue grinder and $250 \mu \mathrm{l}$ Tris Triton lysis buffer (50 mM Tris, $\mathrm{pH} 7.5,100 \mathrm{mM} \mathrm{NaCl}, 2.5 \mathrm{mM} \mathrm{MgCl}, 0.5 \%$ Triton X-100) that included MilliporeSigma protease inhibitors II (P5726) and III (P0044) and a Roche Complete Mini Protease inhibitor tablet (11836170001). Homogenized samples were shaken $1,500 \mathrm{rpm}$ at $4^{\circ} \mathrm{C}$ for $30 \mathrm{~min}-$ utes and then frozen and thawed in liquid nitrogen and $37^{\circ} \mathrm{C} 3$ times, followed by $15,000 \mathrm{~g}$ centrifugation for 10 minutes. $30 \mu \mathrm{g}$ protein was boiled in Laemmli loading buffer and run on a 4\%-20\% Bio-Rad precast gel (5671094). Protein was transferred to a nitrocellulose Bio-Rad Transblot Turbo kit (170-4271). Blots were blocked room temperature for 30 minutes in 5\% milk/PBS-T. Blots were probed overnight at $4^{\circ} \mathrm{C}, 1: 1,000$, with the ATXN1 antibody 11NQ (46) or 12NQ (47) or 1:5,000 Tubulin (MilliporeSigma, A4700). Blots were washed with PBS-T 3 times and then placed in 5\% milk PBS-T plus 1:2,500 species-specific HRP antibodies. (GE NXA931, NA9340V). Blots were washed with PBS-T 3 times, followed by Super Signal West Pico (Thermo, 34078), and imaged on an ImageQuant LAS 4000.

Laser microdissection. One half the cerebellum from each mouse was mounted in Tissue-Tek OCT medium (Sakura Finetek) and frozen using liquid nitrogen. Tissue was cut into $20-\mu \mathrm{m}$ sections and mounted on PEN-Membrane slides (Thermo Fisher Scientific). Mounted tissue was stained using cresyl violet. Subsections of tissue slices were collected using a Leica LMD6500 Laser Dissections Microscope. Isolated tissue samples were transferred to Qiazol lysis buffer (Qiagen) for analysis via RT qPCR and Western blot.

$R T-q P C R$. One half of each brain region from each mouse was homogenized in $500 \mu 1$ Trizol (Thermo Fisher Scientific, 15596026). RNA isolation was done per the manufacture's instructions. cDNA was synthesized in duplicate using $500 \mathrm{ng}$ RNA in $10 \mu \mathrm{l}$ iScript ADV (172-5038) reactions and then diluted 1:5 in water. qPCR was performed using $2 \mu \mathrm{l}$ cDNA in $10 \mu 1$ Roche Probes Master (04707494001) reactions on a Roche 480 Lightcycler. The target gene, mAtxn1, NM_009124 (CACGGTCATTCAGACCACACA, GGTAGCCGATGACAGGAGGTT and probe FAM-CAGCCACGG/ZEN/CCTTCTACGCTGG/3IABKFQ/) and reference gene, Roche mGAPD(05046211001), reactions were amplified in separate wells under cycling conditions: $95^{\circ} \mathrm{C}$ for 10 seconds, $60^{\circ} \mathrm{C}$ for 20 seconds for 35 cycles. $\mathrm{C}_{\mathrm{q}}$ values were determined using the Roche Second Derivative Max calculation. Relative quantification was done using standard $2^{\mathrm{Cq}}$.

Immunofluorescence. Mice were deeply anesthetized and transcardially perfused with $10 \%$ buffered formalin, and 50-micron sections were cut on a vibratome. Epitopes were unmasked by boiling 3 times for 15 seconds each in $0.01 \mathrm{M}$ urea. The sections were blocked for 1 hour in $2 \%$ normal donkey serum, $0.3 \%$ Triton X-100 in 1× PBS. After blocking, the sections were incubated for 48 hours at $4^{\circ} \mathrm{C}$ in blocking buffer with rabbit anti-ASO (1:10,000, Ionis), goat anti-calbindin (1:250, SC-7691, Santa Cruz), and mouse antiNeuN (1:200, MAB377, Millipore). The sections were washed 4 times in PBS and incubated for 24 hours in blocking solution containing secondary antibody. Donkey secondary Alexa Fluor 488, Cy3, and Alexa Fluor 647 were used at 1:500 (Jackson Immunoresearch). Fluorescent images were scanned using an Olympus Fluoview 1000 IX2 inverted microscope.

Behavioral analyses - rotarod. Mice were tested on the rotarod apparatus (Ugo Basile) using an accelerating protocol: 5-50 rpm, 5-minute ramp duration, 5-minute maximum trial length. The test consisted of a total of 4 trials per day for 4 consecutive days. Mice were habituated to the testing room 15 minutes prior to the start of testing on each day. Animals were segregated by sex during testing and run in consistent groups (up to 5 at a time). To ensure enough recovery time between trials, animals were given 10-15 minutes between the end of a trial and the following trial, which included the time to test the other groups in the trial. Trials ended whenever an animal failed to stay on the rotarod or if they made 2 consecutive rotations clinging to the rod and not ambulating. Animals were returned to their home cages after trial completion. The apparatus was cleaned between each animal group within a trial with a $70 \%$ ethanol.

Behavioral analyses - beam walk. The beam walk protocol consisted of 3 consecutive training days, followed by 1 test day. The beam walk apparatus was built in-house by the Mouse Behavior Core using wood and plastic components, consisting of beams (of varying sizes and shapes) that extended to a goal box. 
The apparatus was elevated approximately $50 \mathrm{~cm}$ off a table surface. Testing was performed in a dark room with a single light directed at the start position of the beam to encourage crossing to the goal box. Males and females were segregated during testing. Animals were run at approximately the same time every day and habituated to the behavior room for about 15 minutes prior to the start of training. At the start of the first trial of each day, an animal was placed into the goal box for approximately 15-30 seconds to become familiar before beginning the first trial. After familiarization with the goal box, the mouse was brought to the start end of the beam and placed with the nose right behind the "start" line. Beam walk training (using the 15-mm square beam) consisted of 4 trials per day, with a maximum of 60 seconds allowed for an animal to cross. Animals were tested in smaller groups of 4-7 to allow for approximately 5-minute intertrial intervals. Hind paw foot slips and time(s) to cross the beam were recorded by an investigator blind to treatment and genotype. A foot slip was defined as a hind leg and paw coming completely off the beam in a fast downward sweeping motion. Animals that successfully crossed the beam were left in the goal box for approximately 15 seconds while the beam was wiped down with a $70 \%$ ethanol solution. The animal was then returned to their home cage until the next trial. The goal platform was cleaned with the ethanol solution after each trial and between animals on the first trial of each day. On the test day, 6 beams of decreasing size (either square or round) and increasing difficulty were used with only 2 trials of each. The beam order on test day was (a) $25 \mathrm{~mm}$ (diameter), square; (b) $25 \mathrm{~mm}$, round; (c) $16 \mathrm{~mm}$, square; (d) $19 \mathrm{~mm}$, round; (e) $10 \mathrm{~mm}$, square; and (f) $10 \mathrm{~mm}$, round.

$R N A$ isolation and sequencing. Total RNA was isolated from dissected cerebella (4 samples each of WT vehicle, Sca1 ${ }^{154 \mathrm{Q} / 2 Q}$, and $S c a 1^{154 Q / 2 Q}$ with ASO treatment at 18 weeks), pontes ( 3 samples each at 18 weeks and 4 samples each at 28 weeks of WT vehicle, Sca $1^{154 Q / 2 Q}$, and Sca $1^{154 Q / 2 Q}$ with ASO treatment), and medullae (3 samples each at 18 weeks and 8, 8, and 7 samples, respectively, at 28 weeks of WT vehicle, Sca $1^{154 Q / 2 Q}$, and Sca $1^{154 Q / 2 Q}$ with ASO treatment; see Supplemental Table 1) using TRIzol Reagent (Life Technologies) following the manufacturer's protocols. Tissue was homogenized using RNase-Free Disposable Pellet Pestles (Fisher Scientific) in a motorized chuck. For RNA-seq, RNA was further purified to remove any organic carryover using the RNeasy Mini Kit (Qiagen) following the manufacturer's RNA Cleanup protocol. Purified RNA was sent to the University of Minnesota Genomics Center for quality control, including quantification using fluorimetry (RiboGreen assay, Life Technologies), and RNA integrity was assessed with capillary electrophoresis (Agilent BioAnalyzer 2100, Agilent Technologies Inc.), generating an RNA integrity number (RIN). All submitted samples had greater than $1 \mu \mathrm{g}$ total mass and RINs 7.9 or greater. Library creation was completed using oligo-dT purification of polyadenylated RNA, which was reverse transcribed to create cDNA. cDNA was fragmented, blunt ended, and ligated to barcoded adaptors. Library was size selected to $320 \mathrm{bp} \pm 5 \%$ to produce average inserts of approximately $200 \mathrm{bp}$, and size distribution validated using capillary electrophoresis and quantified using fluorimetry (PicoGreen, Life Technologies) and qPCR. Libraries were then normalized, pooled, and sequenced on an Illumina HiSeq 2000 using a 100-nt paired-end read strategy. Data were stored and maintained on University of Minnesota Supercomputing Institute Servers. Reads were aligned to the mouse reference genome (GRcm38) with hisat2 (48) using default parameters for stranded libraries, the hisat2 GRCm38 index for the genome plus SNPs, and transcripts and using Ensembl's release 87 of the GRCm38 gene annotations. Read counts per gene were summed using featureCounts from the subread package (49). Genes less than about 300 bp are too small to be accurately captured in standard RNA-seq library preparations, so they were discarded from downstream analyses. Differential expression analysis was carried out using the R package DESeq2 (50). The medulla samples were collected and sequenced in two batches, and batch effect was corrected in the model for differential expression analysis. Genes with a FDR value of less than or equal to 0.05 were considered significant. GSEA (v3.0) was used to test for enrichment of Magenta module genes in cerebellum and medulla data using a preranked test. For both data sets, genes were ranked by $-\log _{10}(P$ value $)$ using WT vehicle versus SCA ASO-treated differential expression comparison, with the sign of the fold change assigned to the ranking metric, i.e., if the fold change was negative, the ranking metric was $-1 \times\left(-\log _{10}[P\right.$ value $\left.]\right)$.

WGCNA. Read abundances for 28-week medulla samples were filtered to keep only the 16,344 genes that had at least 50 reads in at least 7 samples (the size of the smallest treatment group) and were then normalized with a variance stabilizing transformation in DESeq2 for WGCNA analysis $(51,52)$. The WGCNA R package (v. 1.51) was used to construct an unsigned gene coexpression network with a soft threshold power [ $\beta$ ] of 15 , which resulted in 13,029 of the genes assigned to 20 coexpression modules. 
All modules were tested for significant association with disease state (WT/vehicle and Sca1 $1^{154 Q / 2 Q} / \mathrm{ASO}$ were considered "healthy" and $S c a 1^{154 Q / 2 Q}$ vehicle was considered "sick") via $t$ test, and $P$ values were adjusted for multiple testing using a Benjamini-Hochberg correction. The Brown module (1,510 genes) was by far the most significantly associated with disease state (adjusted $P=9.077501 \mathrm{E}-08,5$ orders of magnitude smaller than the next most significant module). Data for the Brown module were exported to Cytoscape for visualization. The MEME suite's FIMO tool (v. 5.0.1, ref. 53) was used to detect 2 known Capicua-binding motifs (TGAATGAA and TGAATGGA) in the $2 \mathrm{~kb}$ upstream of the transcription start sites of Brown module genes. These sequences were shuffled and again searched for CIC-binding sites using FIMO; a Fisher's exact test was used to test for enrichment of CIC-binding sites in the original compared with shuffled sequences.

MRS. For MRS analyses, at 5 weeks of age, Atxn $1^{154 Q / 2 Q}$ mice were treated with 1 i.c.v. injection of ASO or vehicle solution; WT C57/B16 littermates received 1 injection of vehicle solution. At 18 ( $N=11-12$ per each of the 3 groups) and 28 weeks $(N=15-16$ per group), mice underwent in vivo, single-voxel MRS of the cerebellum and brainstem at 9.4 tesla (T). Mice used for MRS were housed, 2-4 animals per cage, in a specific pathogen-free facility with a 12-hour-light/dark cycle. They were provided standard food and water ad libitum. MRS data were acquired and analyzed in a blind fashion with respect to the group of animals. For MR scanning, mice were induced with $3 \%$ isoflurane in a 50:50 mixture of $\mathrm{O}_{2} / \mathrm{N}_{2} \mathrm{O}$, and anesthesia was maintained with $1.3 \%-1.5 \%$ isoflurane for the duration of the procedure. Mice were secured in a holder and positioned inside the $9.4 \mathrm{~T}$ magnet for MR measurement. Body temperature was maintained at $37^{\circ} \mathrm{C}$ using a circulating warm water bath and a heating fan controlled by feedback from a fiber-optic rectal thermometer. After each measurement, animals were placed in a recovery cage until they were fully awake before they were returned to their home cage. Following recovery from the final scan, mice were euthanized via carbon dioxide asphyxiation, and brain tissue was harvested for RNA analysis. A quadrature surface coil and a horizontal $9.4 \mathrm{~T}$ magnet (Agilent) were used to acquire MRS measurements according to methods previously described $(11,43)$. VOIs for the MRS measurement were selected using coronal and sagittal images acquired with a rapid acquisition with relaxation enhancement sequence (repetition time $[\mathrm{TR}]=4 \mathrm{~s}$; echo train length $=8$; echo time $(\mathrm{TE})=60 \mathrm{~ms}$; slice thickness $=1 \mathrm{~mm})$. First- and second-order shims were adjusted for each VOI using FASTMAP with echo-planar readout (54). Proton MR spectra were acquired with a shortecho LASER sequence $(55)(\mathrm{TE}=15 \mathrm{~ms} ; \mathrm{TR}=5 \mathrm{~s} ; 128$ transients $)$ from a cerebellar VOI $(2.1 \times 1.8 \times$ $\left.1.8 \mathrm{~mm}^{3} ; 6.8 \mu \mathrm{l}\right)$ and a brainstem VOI $\left(4.3 \times 1.8 \times 1.6 \mathrm{~mm}^{3} ; 12.4 \mu 1\right)$. Spectra were saved as single shots to enable frequency and phase correction. Unsuppressed water spectra were acquired from the same VOI as a metabolite quantification reference. For metabolite quantification, single shots were eddy current, frequency, and phase corrected using MRspa software (http://www.cmrr.umn.edu/downloads/ mrspa/) before summation. Spectra were quantified using LCModel 6.3-0G (56) with the water-scaling option using 19 basis spectra, which were simulated using density matrix formalism (57) based on previously reported chemical shifts and coupling constants $(58,59)$. Concentrations with mean Cramér-Rao lower bounds $\leq 20 \%$ at either 18 or 28 weeks for a given treatment group were reported. Metabolites with strong correlations $(\mathrm{r}<-0.7)$ in the majority of the spectra, specifically $\mathrm{Cr}$ and $\mathrm{PCr}$ as well as Cho and PCho, were reported as the sums of total creatine and tCho. Group means were compared with 1-way ANOVA and the Tukey Method for multiple comparisons.

Statistics. qPCR, RNA-seq, and motor performance data are expressed as mean \pm SEM. Statistical analyses were performed using GraphPad Prism software (version 7.03) and R (version 3.3.3). Significant differences were based on $t$ test, Fisher's exact test, or 1- or 2-way ANOVA, followed by a Bonferroni, Tukey, or Dunnett post hoc test or a Benjamini-Hochberg correction for multiple testing, depending on the analysis (see figure legends). DE genes were determined using DESeq2 (v.1.14.1). $P$ $<0.05$ (or adjusted $P<0.05$, in appropriate cases) was considered as statistically significant. Significance of mouse survival, plotted as Kaplan-Meyer curves, was assessed using log-rank Mantel-Cox and Gehan-Breslow-Wilcoxon tests. Neurochemical profile data are expressed as the mean $\pm 1.96 \times$ SEM (95\% CI). Statistical analyses were performed using GraphPad Prism software (version 7.03). Group means for neurochemical concentrations were compared with 1-way ANOVA, and $P$ values corrected for multiple comparisons using the Tukey Method. $P<0.05$ was considered as statistically significant.

Study approval. The University of Minnesota Institutional Animal Care and Use Committee approved all animal procedures. 


\section{Author contributions}

JF, HBK, GÖ, and HTO conceived the studies. HBK, HYZ, HBC, GÖ, SL, and HTO reviewed the data and wrote and edited the paper. $\mathrm{HBK}$ and EES performed initial ASO screens. JF, BH, and BO performed ASO i.c.v. injections. JF, BO, EES, HPH, CW, LD, ZC, and JPO generated reagents and performed biochemical characterization of mice. MB and TNM performed behavioral analyses. GÖ and SL performed MRS studies and analyzed MRS data. CH performed RNA-seq analyses. OR and PY bred and genotyped all mice.

\section{Acknowledgments}

This work was supported by a grant from the NIH/National Institute of Neurological Disorders and Stroke (NIH/NINDS) (R37 NS022920), a National Ataxia Foundation Pioneer Award, and a Wallin Neuroscience Discovery Award to HTO and a grant from NIH/NINDS (R37NS027699) and the Howard Hughes Medical Institute to HYZ. Additional support was received from the Jay D. Schlueter Ataxia Research Fund. The Center for Magnetic Resonance Research is supported by the National Institute of Biomedical Imaging and Bioengineering (grant P41 EB015894), the Institutional Center Cores for Advanced Neuroimaging (award P30 NS076408), and the W.M. Keck Foundation. The authors wish to thank the Biomedical Genomics Center and Mouse Phenotyping Core at the University of Minnesota. It is with the deepest degree of respect and sadness that the authors dedicate this work to the memory of Jill Friedrich, our colleague and friend, who died in August 2017 from injuries sustained in a biking accident.

Address correspondence to: Harry T. Orr, Department of Laboratory Medicine and Pathology, University of Minnesota, 2101 6th Street SE, Delivery Code 2641B, 3110 WMBB, Minneapolis, Minnesota 55455, USA. Phone: 612.625.3647; Email: orrxx002@umn.edu.

1. Orr HT, et al. Expansion of an unstable trinucleotide CAG repeat in spinocerebellar ataxia type 1. Nat Genet. 1993;4(3):221-226.

2. Genis D, et al. Clinical, neuropathologic, and genetic studies of a large spinocerebellar ataxia type 1 (SCA1) kindred: (CAG)n expansion and early premonitory signs and symptoms. Neurology. 1995;45(1):24-30.

3. Sasaki H, et al. Clinical features and natural history of spinocerebellar ataxia type 1. Acta Neurol Scand. 1996;93(1):64-71.

4. Orengo JP, van der Heijden ME, Hao S, Tang J, Orr HT, Zoghbi HY. Motor neuron degeneration correlates with respiratory dysfunction in SCA1. Dis Model Mech. 2018;11(2):dmm032623.

5. Bürk K, et al. Autosomal dominant cerebellar ataxia type I clinical features and MRI in families with SCA1, SCA2 and SCA3 Brain. 1996;119(Pt 5):1497-1505.

6. Oz G, et al. Neurochemical alterations in spinocerebellar ataxia type 1 and their correlations with clinical status. Mov Disord. 2010;25(9):1253-1261

7. Joers JM, et al. Neurochemical abnormalities in premanifest and early spinocerebellar ataxias. Ann Neurol. 2018;83(4):816-829.

8. Zoghbi HY, Orr HT. Pathogenic mechanisms of a polyglutamine-mediated neurodegenerative disease, spinocerebellar ataxia type 1. J Biol Chem. 2009;284(12):7425-7429.

9. $\mathrm{Zu} \mathrm{T}$, et al. Recovery from polyglutamine-induced neurodegeneration in conditional SCA1 transgenic mice. $J$ Neurosci. 2004;24(40):8853-8861.

10. Oz G, et al. In vivo monitoring of recovery from neurodegeneration in conditional transgenic SCA1 mice. Exp Neurol. 2011;232(2):290-298.

11. Öz G, et al. Assessing recovery from neurodegeneration in spinocerebellar ataxia 1: Comparison of in vivo magnetic resonance spectroscopy with motor testing, gene expression and histology. Neurobiol Dis. 2015;74:158-166.

12. Xia H, et al. RNAi suppresses polyglutamine-induced neurodegeneration in a model of spinocerebellar ataxia. Nat Med. 2004;10(8):816-820.

13. Keiser MS, Boudreau RL, Davidson BL. Broad therapeutic benefit after RNAi expression vector delivery to deep cerebellar nuclei: implications for spinocerebellar ataxia type 1 therapy. Mol Ther. 2014;22(3):588-595.

14. Jafar-Nejad P, Ward CS, Richman R, Orr HT, Zoghbi HY. Regional rescue of spinocerebellar ataxia type 1 phenotypes by 14-3-3epsilon haploinsufficiency in mice underscores complex pathogenicity in neurodegeneration. Proc Natl Acad Sci USA. 2011;108(5):2142-2147.

15. Park J, et al. RAS-MAPK-MSK1 pathway modulates ataxin 1 protein levels and toxicity in SCA1. Nature. 2013;498(7454):325-331.

16. Matilla A, et al. Mice lacking ataxin-1 display learning deficits and decreased hippocampal paired-pulse facilitation. $J$ Neurosci. 1998;18(14):5508-5516.

17. Keiser MS, Kordasiewicz HB, McBride JL. Gene suppression strategies for dominantly inherited neurodegenerative diseases: lessons from Huntington's disease and spinocerebellar ataxia. Hum Mol Genet. 2016;25(R1):R53-R64.

18. Schoch KM, Miller TM. Antisense oligonucleotides: translation from mouse models to human neurodegenerative diseases. Neuron. 2017;94(6):1056-1070.

19. Sztainberg Y, et al. Reversal of phenotypes in MECP2 duplication mice using genetic rescue or antisense oligonucleotides. Nature. 2015;528(7580):123-126.

20. DeVos SL, et al. Tau reduction prevents neuronal loss and reverses pathological tau deposition and seeding in mice with tauopathy. Sci Transl Med. 2017;9(374):eaag0481.

21. Scoles DR, et al. Antisense oligonucleotide therapy for spinocerebellar ataxia type 2. Nature. 2017;544(7650):362-366 
22. McLoughlin HS, et al. Oligonucleotide therapy mitigates disease in spinocerebellar ataxia type 3 mice. Ann Neurol. 2018;84(1):64-77.

23. Finkel RS, et al. Nusinersen versus sham control in infantile-onset spinal muscular atrophy. N Engl J Med. 2017;377(18):1723-1732.

24. Watase K, et al. A long CAG repeat in the mouse Sca1 locus replicates SCA1 features and reveals the impact of protein solubility on selective neurodegeneration. Neuron. 2002;34(6):905-919.

25. Ingram M, et al. Cerebellar transcriptome profiles of ATXN1 transgenic mice reveal SCA1 disease progression and protection pathways. Neuron. 2016;89(6):1194-1207.

26. Lorenzetti D, Watase K, Xu B, Matzuk MM, Orr HT, Zoghbi HY. Repeat instability and motor incoordination in mice with a targeted expanded CAG repeat in the Sca1 locus. Hum Mol Genet. 2000;9(5):779-785.

27. Bennett CF, Swayze EE. RNA targeting therapeutics: molecular mechanisms of antisense oligonucleotides as a therapeutic platform. Annu Rev Pharmacol Toxicol. 2010;50:259-293.

28. Kordasiewicz HB, et al. Sustained therapeutic reversal of Huntington's disease by transient repression of huntingtin synthesis. Neuron. 2012;74(6):1031-1044.

29. Robitaille Y, Schut L, Kish SJ. Structural and immunocytochemical features of olivopontocerebellar atrophy caused by the spinocerebellar ataxia type 1 (SCA-1) mutation define a unique phenotype. Acta Neuropathol. 1995;90(6):572-581.

30. Geary RS. Antisense oligonucleotide pharmacokinetics and metabolism. Expert Opin Drug Metab Toxicol. 2009;5(4):381-391.

31. Reetz K, et al. Genotype-specific patterns of atrophy progression are more sensitive than clinical decline in SCA1, SCA3 and SCA6. Brain. 2013;136(Pt 3):905-917.

32. Subramanian A, et al. Gene set enrichment analysis: a knowledge-based approach for interpreting genome-wide expression profiles. Proc Natl Acad Sci USA. 2005;102(43):15545-15550.

33. Zhang B, Horvath S. A general framework for weighted gene co-expression network analysis. Stat Appl Genet Mol Biol. 2005;4:Article17.

34. Parikshak NN, Gandal MJ, Geschwind DH. Systems biology and gene networks in neurodevelopmental and neurodegenerative disorders. Nat Rev Genet. 2015;16(8):441-458.

35. Hu Y, Pan S, Zhang HT. Interaction of Cdk5 and cAMP/PKA signaling in the mediation of neuropsychiatric and neurodegenerative diseases. Adv Neurobiol. 2017;17:45-61.

36. Rousseaux MWC, et al. ATXN1-CIC complex is the primary driver of cerebellar pathology in spinocerebellar ataxia type 1 through a gain-of-function mechanism. Neuron. 2018;97(6):1235-1243.e5.

37. Al-Ramahi I, et al. High-throughput functional analysis distinguishes pathogenic, nonpathogenic, and compensatory transcrip tional changes in neurodegeneration. Cell Syst. 2018;7(1):28-40.e4.

38. Cudalbu C, et al. The C57BL/6J mouse exhibits sporadic congenital portosystemic shunts. PLoS One. 2013;8(7):e69782.

39. Emir UE, Brent Clark H, Vollmers ML, Eberly LE, Öz G. Non-invasive detection of neurochemical changes prior to overt pathology in a mouse model of spinocerebellar ataxia type 1. J Neurochem. 2013;127(5):660-668.

40. Wu JY, Prentice H. Role of taurine in the central nervous system. J Biomed Sci. 2010;17 Suppl 1:S1.

41. Oz G, et al. Clinical proton MR spectroscopy in central nervous system disorders. Radiology. 2014;270(3):658-679.

42. Fryer JD, et al. Exercise and genetic rescue of SCA1 via the transcriptional repressor Capicua. Science. 2011;334(6056):690-693

43. Oz G, et al. Noninvasive detection of presymptomatic and progressive neurodegeneration in a mouse model of spinocerebellar ataxia type 1. J Neurosci. 2010;30(10):3831-3838.

44. Mountford CE, Stanwell P, Lin A, Ramadan S, Ross B. Neurospectroscopy: the past, present and future. Chem Rev. 2010;110(5):3060-3086.

45. Ross BM, et al. Abnormal activity of membrane phospholipid synthetic enzymes in the brain of patients with Friedreich's ataxia and spinocerebellar atrophy type-1. Mov Disord. 2000;15(2):294-300.

46. Servadio A, Koshy B, Armstrong D, Antalffy B, Orr HT, Zoghbi HY. Expression analysis of the ataxin-1 protein in tissues from normal and spinocerebellar ataxia type 1 individuals. Nat Genet. 1995;10(1):94-98

47. Pérez Ortiz JM, et al. Reduction of protein kinase A-mediated phosphorylation of ATXN1-S776 in Purkinje cells delays onset of Ataxia in a SCA1 mouse model. Neurobiol Dis. 2018;116:93-105.

48. Kim D, Langmead B, Salzberg SL. HISAT: a fast spliced aligner with low memory requirements. Nat Methods. 2015;12(4):357-360

49. Liao Y, Smyth GK, Shi W. featureCounts: an efficient general purpose program for assigning sequence reads to genomic features. Bioinformatics. 2014;30(7):923-930.

50. Love MI, Huber W, Anders S. Moderated estimation of fold change and dispersion for RNA-seq data with DESeq2. Genome Biol. 2014;15(12):550.

51. Langfelder P, Horvath S. WGCNA: an R package for weighted correlation network analysis. BMC Bioinformatics. 2008;9:559.

52. Langfelder P, Horvath S. Fast R functions for robust correlations and hierarchical clustering. J Stat Softw. 2012;46(11):i11.

53. Grant CE, Bailey TL, Noble WS. FIMO: scanning for occurrences of a given motif. Bioinformatics. 2011;27(7):1017-1018

54. Gruetter R, Tkác I. Field mapping without reference scan using asymmetric echo-planar techniques. Magn Reson Med. 2000;43(2):319-323.

55. Garwood M, DelaBarre L. The return of the frequency sweep: designing adiabatic pulses for contemporary NMR. JMagn Reson. 2001;153(2):155-177.

56. Provencher SW. Automatic quantitation of localized in vivo 1H spectra with LCModel. NMR Biomed. 2001;14(4):260-264.

57. Deelchand DK, Henry PG, Uğurbil K, Marjanska M. Measurement of transverse relaxation times of J-coupled metabolites in the human visual cortex at 4 T. Magn Reson Med. 2012;67(4):891-897.

58. Govindaraju V, Young K, Maudsley AA. Proton NMR chemical shifts and coupling constants for brain metabolites. NMR Biomed. 2000;13(3):129-153.

59. Tkac I, Refinement of simulated basis set for LCModel analysis. 16th Scientific Meeting of the ISMRM. Toronto, Canada $2008 ;$ p. 1624 